\title{
Application of Iterative Robust Model-based Optimal Experimental Design for the Calibration of Biocatalytic Models
}

Van Daele, Timothy; Gernaey, Krist V.; Ringborg, Rolf Hoffmeyer; Börner, Tim; Heintz, Søren; Van Hauwermeiren, Daan; Grey, Carl; Krühne, Ulrich; Adlercreutz, Patrick; Nopens, Ingmar

\author{
Published in: \\ Biotechnology Progress \\ Link to article, DOI: \\ 10.1002/btpr.2515 \\ Publication date: \\ 2017 \\ Document Version \\ Peer reviewed version \\ Link back to DTU Orbit
}

Citation (APA):

Van Daele, T., Gernaey, K. V., Ringborg, R. H., Börner, T., Heintz, S., Van Hauwermeiren, D., Grey, C., Krühne, U., Adlercreutz, P., \& Nopens, I. (2017). Application of Iterative Robust Model-based Optimal Experimental Design for the Calibration of Biocatalytic Models. Biotechnology Progress, 33(5), 1278-1293 . https://doi.org/10.1002/btpr.2515

\section{General rights}

Copyright and moral rights for the publications made accessible in the public portal are retained by the authors and/or other copyright owners and it is a condition of accessing publications that users recognise and abide by the legal requirements associated with these rights.

- Users may download and print one copy of any publication from the public portal for the purpose of private study or research.

- You may not further distribute the material or use it for any profit-making activity or commercial gain

- You may freely distribute the URL identifying the publication in the public portal 


\title{
Application of Iterative Robust Model-based Optimal Experimental Design for the Calibration of Biocatalytic Models
}

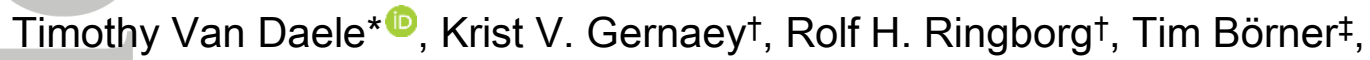
Søren Heintz`, Daan Van Hauwermeiren*, Carl Grey ${ }^{\ddagger}$, Ulrich Krühne†, Patrick Adlercreutz $\ddagger$, Ingmar Nopens *§

*BIOMATH, Department of Mathematical Modelling, Statistics and

Bioinformatics, Faculty of Bioscience Engineering, Ghent University, Coupure

Links 653, 9000 Ghent, Belgium

Corresponding Author: Ingmar.Nopens@ugent.be

tProcess and Systems Engineering Center (PROSYS), Department of

Chemical and Biochemical Engineering, Technical University of Denmark,

Building 229, 2800 Kgs. Lyngby, Denmark

‡Department of Biotechnology, Chemical Center, Lund University, P.O. Box

124, S-211 00 Lund, Sweden

§Corresponding author: ingmar.nopens@ugent.be

Abstract-The aim of model calibration is to estimate unique parameter values from available experimental data, here applied to a biocatalytic process. The traditional approach of first gathering data followed by performing a model calibration is inefficient, since the information gathered during experimentation is not actively used to optimise the experimental design. By applying an iterative robust model-based optimal experimental design, the limited amount of data collected is used to design additional informative experiments. The algorithm is used here to calibrate the initial reaction rate of an $\omega$-transaminase catalysed reaction in a more accurate way. The parameter confidence region estimated from the Fisher Information Matrix is compared with the likelihood confidence region, which is a more accurate, but also a computationally more expensive method. As a result, an important deviation between both approaches is found, confirming that linearisation methods should be applied with care for nonlinear models.

Keywords: Biocatalysis, robust model-based optimal experimental design, $\omega$ transaminase, curvature, Fisher information matrix

\section{Nomenclature}

Abbreviations

ACE acetone

This article has been accepted for publication and undergone full peer review but has not been through the copyediting, typesetting, pagination and proofreading process which may lead to differences between this version and the Version of Record. Please cite this article as doi: $10.1002 / b t p r .2515$

(c) 2017 American Institute of Chemical Engineers Biotechnol Prog

Received: Oct 14, 2017; Revised: May 08, 2017; Accepted: J un 09, 2017 
BA benzylacetone

CFD computational fluid dynamics

$\mathrm{Cl}$ confidence interval

DOF degree of freedom

IPA isopropylamine

MPPA 1-methyl-3-phenylpropylamine

OED optimal experimental design

PLP pyridoxal 5'-phosphate

rMbOED robust Model-based OED

SD standard deviation

\section{Model Parameters \& Variables}

$\left[E_{\mathrm{t}}[\mathrm{U} / \mathrm{mL}]\right.$ total enzyme concentration

$K_{\text {ACE }}[\mathrm{mM}]$ Michaelis constant ACE

$K_{\mathrm{BA}}[\mathrm{mM}]$ Michaelis constant BA

$K_{\text {eq }}[-]$ equilibrium constant

$K_{\text {ilPA }}[\mathrm{mM}]$ dissociation constant IPA

$K_{\text {iMPPA }}[\mathrm{mM}]$ dissociation constant MPPA

$K_{\text {IPA }}[\mathrm{mM}]$ Michaelis constant IPA

$\mathrm{K}_{\text {MPPA }}[\mathrm{mM}]$ Michaelis constant MPPA

$V_{\mathrm{f}}[\mathrm{nmol} /($ Umin) $] \max$ forward reaction rate

$V_{\mathrm{r}}[\mathrm{nmol} /($ Umin) $]$ max backward reaction rate

$v[\mathrm{nmol} /(\mathrm{mLmin})]$ reaction rate 


\section{Symbols}

$\lambda$ Eigenvalue

FIM Fisher information matrix

H Hessian matrix

$\mathbf{x}$ independent variables

$\mathbf{X}$ design space

J objective function

$\Sigma$ measurement error covariance matrix

$\theta$ parameter set

$\Theta$ parameter space

$\Phi$ parameter estimates error covariance matrix

Q weight matrix

y measurements

$\hat{y}$ measurable model outputs

\section{Introduction}

Biocatalysts convert substrates to products of interest at a certain rate, which depends on the local environmental conditions. The relation between the reaction rate and the local environmental conditions can be formalised in a mathematical model. A well-known and widely used mathematical model is the Michaelis-Menten model (Equation 1), which describes the irreversible conversion of a substrate to a product by a biocatalyst.

$v=\frac{V_{\max }[S]}{K_{\mathrm{m}}+[S]}$ 
where $v$ denotes the reaction rate, $[S]$ the substrate concentration, $K_{m}$ the Michaelis constant, and $V_{\text {max }}$ the maximum reaction rate (which is dependent on the total enzyme concentration). If $[S]=K_{\mathrm{m}}$, the reaction rate $v$ equals half of the maximum reaction rate $V_{\max }$. The two parameters describing the relation between the reaction rate and the substrate concentration $\left(V_{\max }\right.$ and $K_{\mathrm{m}}$ depend on the substrate and enzyme used, but also on conditions such as the temperature and the $\mathrm{pH}$. Consequently, these parameters need to be " updated" for each specific case. In most cases, experimental data are collected at certain conditions allowing to estimate the actual parameter values. In the literature, different approaches to estimate model parameters are available and this remains an important aspect of the modelling exercise. Two major classes of methods can be distinguished, i.e. the linear plotting and nonlinear regression [10]. The linear plotting methods are based on algebraic expressions of simplified kinetic differential equations at initial rate conditions to give a series of straight line equations. Different linear plotting methods exist [10]: Lineweaver-Burk [33], Hanes-Woolf [27], Eadie-Hofstee $[\underline{20}, \underline{28}$, the direct linear plot [21], and the Dixon plot [16] (which is used in particular for determining enzyme inhibition constants). It might seem that the choice of linear transformation is unimportant, since they are all variants of the same equation, and thus would yield an equal accuracy. However, this is only true if both the concentrations and measurements would be errorless [19]. By transforming the equation, the error distribution is distorted depending on the kind of transformation or linearisation that is applied [37]. Dowd and Riggs [19] compared the accuracy of the Lineweaver-Burk plot, Hanes-Woolf plot, and Eadie-Hofstee plot and found that closeness of fit is always the best for the Lineweaver-Burk plot and worst for the Eadie-Hofstee. However, the accuracy with which the Michaelis constants were estimated is the greatest for Eadie-Hofstee and worst for the Lineweaver-Burk plot, leading to the paradox that the "worst fitting" line was yielding the "best" parameter estimates and vice versa. Dowd and Riggs [19] stated that the popularity of the Lineweaver-Burk method may be based upon the ability to provide what seems a good fit even when the experimental data are poor. In the original paper, Lineweaver and Burk [33] already stated that "the relative weighting of 
the experimental observations alters in a definite matter when the form of the equation is altered, and if not taken into account may alter slightly the parameter constants obtained'. By applying the proper weighting, identical parameter estimates can be obtained as the Eadie-Hofstee approach. However, Dowd and Riggs [19] stated that calculating the proper weighting factors is inconvenient, and often coupled with ignorance. Ranaldi et al. [42] extended the analysis of Dowd and Riggs [19] by also including the direct linear plot and the nonlinear regression. Nonlinear regression, also known as nonlinear optimisation, reduces the offset between the model and data using an objective function, without the need for linearising the model. Ranaldi et al. [42] showed that using nonlinear regression, even without using the proper weighting factors, yields the most reliable estimates for the different parameter values. Linear methods are still useful as graphical methods, but not as quantitative methods to estimate parameter values.

It is clear from the above that linear plotting methods should be omitted to estimate parameter values from experimental data. However, this is only one step in the entire parameter estimation approach. The experiments, which were designed to gather the necessary data, had to be designed first. Traditionally, first all the experimental data are gathered before starting the parameter estimation exercise $[\underline{29}, 1]$, whereby the gathered information is not used during the experimentation phase to adjust experimental conditions (i.e. experimentation and model calibration are conducted sequentially). This seems abstract, but can be easily illustrated using the Michaelis-Menten model (Equation 1). Let us assume, that initially no information is available about the parameter values, and thus a proper experimental design cannot be set up. Therefore, one first has to perform experiments before trying to estimate the parameter values. However, if all experiments are designed in the region where $\left[S{ }^{2}<K_{\mathrm{m}}\right.$, Equation 1 reduces to $v=V_{\max }[S] / K_{\mathrm{m}}$. In this way, only the ratio between the two parameters can be estimated, and thus more experiments need to be performed. Measuring additional conversion rates at concentrations well above $K_{\mathrm{m}}$ results in an estimate for $V_{\max }$, and thus allows to estimate both parameters in a reliable way. To avoid this kind of 
identification problems, i.e. lack of informative data, it may be more interesting to use an iterative approach as depicted in Figure 1. Dochain and Vanrolleghem [17] and Goujot et al. [24] proposed this procedure in order to optimise the experimental conditions. First, some preliminary experiments are conducted, which can afterwards be used to perform an initial parameter estimation. Next, one needs to assess whether the reliability of the parameter estimates is sufficiently high. If not, new informative experiments need to be conducted to gain additional information. Using the model, regions with high information content can be detected and new experiments will be designed. These new experiments are then performed in the lab, and afterwards this new information can be added and used for improving the quality of the parameter estimates. Application of this strategy is powerful and is often referred to as iterative Optimal Experimental Design (OED) [17, 24]. The effectivity of these OED methods is highly dependent on the accuracy of the initial parameter estimates. Therefore more robust OED methods have been developed which are less sensitive to these parameter estimates. Despite the fact that these robust OED procedures are available, the nonrobust OED methods are applied more frequently in literature $[\underline{24}, \underline{35}, \underline{3}]$.

In the following sections, the reaction under study and the corresponding model to be calibrated will be discussed. Next, some theoretical background will be given about parameter confidence estimation, and how this can be used to design informative experiments. Next, the maximin algorithm, which is a robust OED procedure, is applied for this specific case. Finally, the confidence region of the parameter estimates is calculated using the traditional approach of the Fisher Information Matrix and compared with the likelihood method, which is a more accurate, but computationally more expensive approach.

\section{Theoretical background}

\section{Reaction}

The reaction under study is the synthesis of acetone (ACE) and 1-methyl-3phenylpropylamine (MPPA) from isopropylamine (IPA) and benzylacetone 
(BA) by means of an $\omega$-transaminase ( $\omega-\mathrm{TA})$. By using $\omega$-transaminase (EC 2.6.1.X), optically pure chiral amines are produced by transferring the amine group from an amine donor, to a pro-chiral acceptor ketone, yielding a chiral amine and a ketone as co-product (Figure 2). The enzyme requires pyridoxal 5'-phosphate (PLP) as a cofactor to act as a shuttle to transfer the amine moiety between the molecules $[\underline{1}, \underline{31}]$.

\section{Kinetic Model}

The reaction in Figure 2 obeys the ping-pong bi-bi mechanism (also known as substituted-enzyme mechanism) [11]. The quasi steady-state model of the plain ping-pong bi-bi mechanism, i.e. without substrate or product inhibition, is given in Equation 2 [44].

$$
\begin{gathered}
v=\frac{V_{\mathrm{f}} V_{\mathrm{r}}\left([I P A][B A]-\frac{[A C E][M P P A]}{K_{\mathrm{eq}}}\right)}{V_{\mathrm{r}} K_{\mathrm{BA}}[I P A]+V_{\mathrm{r}} K_{\mathrm{IPA}}[B A]+V_{\mathrm{r}}[I P A][B A]+\frac{V_{\mathrm{f}} K_{\mathrm{MPPA}}[A C E]}{K_{\mathrm{eq}}}+\frac{V_{\mathrm{f}} K_{\mathrm{ACE}}[M P P A]}{K_{\mathrm{eq}}}} \\
+\frac{V_{\mathrm{f}}[A C E][M P P A]}{K_{\mathrm{eq}}}+\frac{V_{\mathrm{f}} K_{\mathrm{MPPA}}[I P A][A C E]}{K_{\mathrm{eq}} K_{\mathrm{iPA}}}+\frac{V_{\mathrm{r}} K_{\mathrm{IPA}}[B A][M P P A]}{K_{\mathrm{iMPA}}}
\end{gathered}
$$

where $[I P A],[B A],[A C E]$, and $[M P P A]$ represent the substrate/product concentrations [mM], $V_{\mathrm{f}}$, and $V_{\mathrm{r}}$ the maximum forward and backward reaction rate [nmol/(Umin)], and $K_{\mathrm{BA}}, K_{\mathrm{IPA}}, K_{\mathrm{MPPA}}, K_{\mathrm{ACE}}$ the Michaelis constants [mM]. $K_{\text {eq }}$ represents the equilibrium constant [-] and $K_{\text {ilPA }}$ and $K_{\text {iMPPA }}$ represent the dissociation constants [mM] [11, 44]. Equation 2 contains 9 kinetic parameters, which all have to be estimated from experimental data. However, by using the Haldane relationship, $K_{\text {eq }}$ can be estimated from the other parameter values. The Haldane relationship for a plain ping-pong bi-bi mechanism is given in Equation 3 [ㄴ4, $\underline{45}]$.

$$
K_{\mathrm{eq}}=\left(\frac{V_{\mathrm{f}}}{V_{\mathrm{r}}}\right)^{2} \frac{K_{\mathrm{ACE}} K_{\mathrm{MPPA}}}{K_{\mathrm{IPA}} K_{\mathrm{BA}}}
$$


By using this relationship, the total number of kinetic parameters which need to be estimated is reduced to 8 .

\section{Parameter estimation}

As previously discussed, different approaches exist to estimate the kinetic parameter values. However, it can be challenging to calibrate all eight parameters of Equation 2. Chen et al. [10] and Al-Haque et al. [1] proposed a methodology to reduce the number of parameters which need to be estimated simultaneously by reducing the full model to several simpler initial rate models. For example at very low product concentrations, Equation 2 can be reduced to the initial forward reaction rate $v_{\text {forw }}$ in Equation 4 . In this way, only three parameters need to be estimated simultaneously under these conditions.

$$
v_{\text {forw }}=\frac{V_{\mathrm{f}}[I P A][B A]}{K_{\mathrm{BA}}[I P A]+K_{\mathrm{IPA}}[B A]+[I P A][B A]}
$$

Similarly, at very low substrate concentrations, Equation 2 can be reduced to the initial backward reaction rate $v_{\text {back }}$ (Equation 5).

$$
v_{\text {back }}=\frac{V_{\mathrm{r}}[M P P A][A C E]}{K_{\mathrm{ACE}}[M P P A]+K_{\mathrm{MPA}}[A C E]+[M P P A][A C E]}
$$

After calibrating both Equations 4 and 5, only two parameters remain to be calibrated in the original rate Equation 2, i.e. both dissociation constants $K_{\text {iPA }}$ and $K_{\text {iMPPA. }}$. Al-Haque et al. [1] proposed to use progress curves at different substrate and product concentrations to estimate the dissociation constants and $K_{\text {eq. }}$. However, in this paper initial rate experiments, which are spiked with product, will be used to calibrate the dissociation constants since such data were already available $(B)$. To ensure that the parameter estimates are suitable, progress curve analysis will be used to validate the full model under different conditions.

The parameter values are estimated by means of nonlinear regression. The weighted sum of squared errors is used as a cost function. 


$$
J(\boldsymbol{\theta})=\sum_{i=1}^{N}\left(\mathbf{y}_{i}-\hat{\mathbf{y}}\left(\mathbf{x}_{i}, \boldsymbol{\theta}\right)\right)^{\top} \mathbf{Q}_{i}\left(\mathbf{y}_{i}-\hat{\mathbf{y}}\left(\mathbf{x}_{i}, \boldsymbol{\theta}\right)\right)
$$

where $J$ represents the cost function which is function of the parameter estimate $\boldsymbol{\theta} . \mathrm{y}$ represents an $N \times M$ matrix containing the $M$ measurements for $N$ samples. $\hat{\mathbf{y}}$ also represent a $N \times M$ matrix containing the measurable $M$ model predictions for the $N$ samples. $\mathrm{Q}$ is a $N \times M \times M$ matrix containing user-supplied weighting coefficients. Typically, $\mathbf{Q}$ is chosen as the inverse of the measurement error covariance matrix $\Sigma[35,39]$. The diagonal of $\Sigma$ contains the variances of $\mathbf{y}$, the off-diagonal elements represent the covariances between the different measurements. In this way, the measurement uncertainty is inherently incorporated in Equation 6, and resulting in Equation 7 [49].

$$
J(\boldsymbol{\theta})=\sum_{i=1}^{N}\left(\mathbf{y}_{i}-\hat{\mathbf{y}}\left(\mathbf{x}_{i}, \boldsymbol{\theta}\right)\right)^{\top} \boldsymbol{\Sigma}_{i}^{-1}\left(\mathbf{y}_{i}-\hat{\mathbf{y}}\left(\mathbf{x}_{i}, \boldsymbol{\theta}\right)\right)
$$

By using nonlinear optimisation, the optimal parameter estimate $\theta$ can be found by minimising the objective function $J$. Different optimisation techniques exist, which can be divided in two major classes: local and global optimisation algorithms [17]. As the name suggests, local optimisation algorithms try to find the minimal value of $J$ in a local neighbourhood starting from an initial guess in the parameter space. However, when the objective function contains multiple local minima and one global minimum, it is likely that the local optimisation algorithm will not end up in the global minimum. To circumvent this problem, global optimisation algorithms are available which are less sensitive to these local minima, though typically are computationally more expensive. However, it is important to be aware that the application of these global algorithms cannot guarantee that the final minimum is the global minimum. In this paper, the parameter estimation is performed using the downhill simplex algorithm [38], which is a local optimisation method. However, the downhill simplex algorithm was repeated a number of times for different initial parameter guesses to avoid ending up in a local minimum.

\section{Confidence regions}


After finishing a (preliminary) model calibration, there is still the need to assess the quality of these parameter estimates, an often forgotten or ignored step in parameter estimation. Some parameters might have little or no influence on the model output, and therefore the estimated parameter value will be meaningless. Uncertain parameter estimates indicate that the available data are not sufficiently informative to extract information with regard to that parameter. If the collection of informative data is unfeasible, the model can be regarded as overparameterised and should be adapted in order to yield reliable predictions [9, 14]. In this context, Marsili-Libelli et al. [35] stated that parameter values always need to be accompanied by a confidence region in order to be meaningful. The confidence region is the region in which it can be expected that, with a certain probability, the true parameters will lie [35]. An " exact" confidence region, in the sense that it is not based on any approximations, is given by Equation 8 [43]:

$\{\boldsymbol{\theta}: J(\boldsymbol{\theta}) \leq c J(\boldsymbol{\theta})\}$,

where $c>1$. The constant $c$ is generally unknown, but can be approximated for a sufficiently large number of data points $N[\underline{7}, \underline{43}]$ :

$\left\{\boldsymbol{\theta}: J(\boldsymbol{\theta}) \leq\left(1+\frac{P}{N-P} F_{P, N-P}^{\alpha}\right) J(\boldsymbol{\theta})\right\}$,

where $F_{P, N-P}^{\alpha}$ is the upper $\alpha$ critical level of the $F$-distribution with $P$ (number of estimated parameters) and $N-P$ degrees of freedom. The confidence region produced by Equation 9 , is generally referred to as the likelihood confidence region. The expectation surface of the objective function is planar if there exists a reparametrisation of $\hat{\mathbf{y}}\left(\mathbf{x}_{i}, \boldsymbol{\theta}\right)$ that makes the function linear in the $P$ parameters [18]. If this is the case, the confidence regions constructed by the likelihood method are exact. One advantage of using the likelihood method is that the confidence region is not affected by any reparametrisation of the function $\hat{\mathbf{y}}$. However, to obtain the likelihood confidence region, a lot of calculations need to be performed since $J$ needs to be evaluated at a sufficient number of points to produce a contour [18]. To overcome this 
computational burden, most often linear approximations of the objective function $J$ are used to construct the confidence region. The objective function $J(\boldsymbol{\theta})$ (Equation 7) can be approximated by a second order Taylor series:

$$
J(\boldsymbol{\theta}) \approx J(\boldsymbol{\theta})+\frac{\partial J(\boldsymbol{\theta})}{\partial \boldsymbol{\theta}}(\boldsymbol{\theta}-\boldsymbol{\theta})+\frac{1}{2}\left[(\boldsymbol{\theta}-\boldsymbol{\theta})^{\top} \frac{\partial^{2} J(\boldsymbol{\theta})}{\partial \boldsymbol{\theta} \partial \boldsymbol{\theta}^{\top}}(\boldsymbol{\theta}-\boldsymbol{\theta})\right]
$$

In a (global) minimum, the first derivative of the objective function $J$ equals zero, reducing Equation 10 to Equation 11.

$$
J(\boldsymbol{\theta}) \approx J(\boldsymbol{\theta})+\frac{1}{2}\left[(\boldsymbol{\theta}-\boldsymbol{\theta})^{\top} \frac{\partial^{2} J(\boldsymbol{\theta})}{\partial \boldsymbol{\theta} \partial \boldsymbol{\theta}^{\top}}(\boldsymbol{\theta}-\boldsymbol{\theta})\right]
$$

Substituting Equation 11 in Equation 9 yields a new expression for the confidence region:

$(\boldsymbol{\theta}-\boldsymbol{\theta})^{\top} \boldsymbol{\Phi}^{-1}(\boldsymbol{\theta})(\boldsymbol{\theta}-\boldsymbol{\theta}) \leq P F_{P, N-P}^{\alpha}$

where $\boldsymbol{\Phi}^{-1}(\boldsymbol{\theta})$ is the inverse of the parameter estimation error covariance matrix defined by [35]:

$$
\boldsymbol{\Phi}(\boldsymbol{\theta})=2 \frac{J(\boldsymbol{\theta})}{N-P} \mathbf{H}^{-1}(\boldsymbol{\theta})
$$

where $J(\theta) /(N-P)$ is an approximation of the residual variance $s^{2}$. For a sufficiently large number of samples and in case of a perfect model fit, $s^{2}$ will approximate the real variance $\sigma^{2}$ of the normal error distribution. This approximation is based on the fact that, assuming the model is correct, the residuals will be random errors and the average of these squared residuals is an estimate of the error variance. In the above equation, $\mathbf{H}^{-1}(\boldsymbol{\theta})$ is the inverse Hessian matrix defined by:

$$
\mathbf{H}=\left.\frac{\partial^{2} J}{\partial \boldsymbol{\theta} \partial \boldsymbol{\theta}^{\top}}\right|_{\theta}
$$

According to the Cramér-Rao inequality [34], the inverse of the $\mathrm{H}$ is equal to the lower bound of the parameter estimation error covariance matrix $\boldsymbol{\Phi}$ if the 
measurement errors are independent samples taken from a normal distribution with zero mean. The linear approximation of the confidence region (Equation 12) is only exact for linear models. In this case the objective function contours are of a quadratic form, meaning that the two-dimensional confidence regions are ellipses and the three-dimensional confidence regions are ellipsoids. However, for nonlinear models Equation 7 is not exactly quadratic, and as a result the linear approximation of Equation 12 is only appropriate if the curvature of the model (i.e. second derivative of $\hat{\mathbf{y}}$ to the parameters $\boldsymbol{\theta}$ ) is sufficiently small. In much of the applied literature $[1, \underline{49}, \underline{24}]$, the importance of the curvature is ignored. Bates and Watts [ㄷ] proposed relative curvature measures which allow to determine whether the model nonlinearity is important. These curvature measures can be divided in two kinds of curvatures, i.e. the intrinsic curvature and the parameter-effects curvature. The intrinsic curvature measures how much the expectation surface deviates from a plane [8]. The parameter-effects curvature represents the degree of curvature induced by the choice of the parameters and its parametrisation. Bates and Watts [4] found that the nonlinearity induced by the parametrisation is generally greater than the intrinsic nonlinearity of the model. Donaldson and Schnabel [18] confirmed that the parameter-effects curvature provides an excellent indication when the linearisation method may produce less satisfactory results. Therefore, these relative curvature measures will be used to determine whether the linear approximation of the confidence region can be regarded as reliable.

\section{Parameter estimate uncertainty and correlation}

From the parameter estimation error covariance matrix (Equation 15), the parameter uncertainty and correlation can be obtained.

$$
\boldsymbol{\Phi}=\left[\begin{array}{cccc}
\sigma_{\theta_{1}}^{2} & \operatorname{Cov}\left(\theta_{1}, \theta_{2}\right) & \ldots & \operatorname{Cov}\left(\theta_{1}, \theta_{P}\right) \\
\operatorname{Cov}\left(\theta_{2}, \theta_{1}\right) & \sigma_{\theta_{2}}^{2} & & \\
\vdots & & \ddots & \\
\operatorname{Cov}\left(\theta_{P}, \theta_{1}\right) & & & \sigma_{\theta_{P}}^{2}
\end{array}\right]
$$


The diagonal elements of this matrix represent the variances $\left(\sigma_{\theta_{i}}^{2}\right)$ of the errors of the parameter estimates. The off-diagonal elements represent the covariances between the parameter estimation errors. Based on these variances and covariances, the elements of the linear correlation matrix can be calculated as:

$\rho_{\theta_{i}, \theta_{j}}=\frac{\operatorname{Cov}\left(\theta_{i}, \theta_{j}\right)}{\sqrt{\sigma_{\theta_{i}}^{2} \sigma_{\theta_{j}}^{2}}}$

As mentioned before, the parameter estimation error covariance matrix $\Phi$ can be used to construct the confidence regions. The confidence interval $\delta_{\theta_{i}}$ of a single parameter $\hat{\theta}_{i}$ is typically calculated as [43]:

$\delta_{\theta_{i}}=\sqrt{\sigma_{\theta_{i}}^{2}} t_{N-P}^{\alpha}$,

where $t_{N-P}^{\alpha}$ is the two-tailed Student t-distribution for the given confidence level $\alpha$ and $N-P$ degrees of freedom.

As stated in Equation 13, the parameter estimation error covariance matrix $\mathbf{\Phi}$ can be related to the Hessian matrix $\mathrm{H}$ in Equation 14. However, the parameter estimation error covariance matrix $\boldsymbol{\Phi}$ is most often calculated from the so-called Fisher Information Matrix (FIM):

$$
\boldsymbol{F I M}(\boldsymbol{\theta}, \mathbf{x})=\sum_{i=1}^{N}\left(\left.\frac{\partial \hat{\mathbf{y}}\left(\mathbf{x}_{i}, \boldsymbol{\theta}\right)}{\partial \boldsymbol{\theta}}\right|_{\theta}\right)^{\top} \boldsymbol{\Sigma}_{i}^{-1}\left(\left.\frac{\partial \hat{\mathbf{y}}\left(\mathbf{x}_{i}, \boldsymbol{\theta}\right)}{\partial \boldsymbol{\theta}}\right|_{\theta}\right)
$$

The Fisher Information Matrix FIM is an approximation of the Hessian matrix $\mathrm{H}$, and the relation between both matrices is given by $[\underline{40}, \underline{17}, \underline{35}]$ : 


$$
\begin{aligned}
& \mathbf{H}=\left.\frac{\partial^{2} \boldsymbol{J}}{\partial \boldsymbol{\theta} \partial \boldsymbol{\theta}^{\top}}\right|_{\boldsymbol{\theta}} \\
& =\frac{\partial}{\partial \boldsymbol{\theta}} \frac{\partial}{\partial \boldsymbol{\theta}^{\top}}\left[\sum_{i=1}^{N}\left(\mathbf{y}_{i}-\hat{\mathbf{y}}\left(\mathbf{x}_{i}, \boldsymbol{\theta}\right)\right)^{\top} \boldsymbol{\Sigma}_{i}^{-1}\left(\mathbf{y}_{i}-\hat{\mathbf{y}}\left(\mathbf{x}_{i}, \boldsymbol{\theta}\right)\right)\right] \\
& =2 \sum_{i=1}^{N}\left[\left(\left.\frac{\partial \hat{\mathbf{y}}\left(\mathbf{x}_{i}, \boldsymbol{\theta}\right)}{\partial \boldsymbol{\theta}}\right|_{\boldsymbol{\theta}}\right)^{\top} \boldsymbol{\Sigma}_{i}^{-1}\left(\left.\frac{\partial \hat{\mathbf{y}}\left(\mathbf{x}_{i}, \boldsymbol{\theta}\right)}{\partial \boldsymbol{\theta}}\right|_{\theta}\right)-\left(\mathbf{y}_{i}-\hat{\mathbf{y}}\left(\mathbf{x}_{i}, \boldsymbol{\theta}\right)\right)^{\top} \boldsymbol{\Sigma}_{i}^{-1}\left(\left.\frac{\partial^{2} \hat{\mathbf{y}}\left(\mathbf{x}_{i}, \boldsymbol{\theta}\right)}{\partial \boldsymbol{\theta} \partial \boldsymbol{\theta}^{\top}}\right|_{\theta}\right)\right] \\
& =2 \sum_{i=1}^{N}\left[\left(\left.\frac{\partial \hat{\mathbf{y}}\left(\mathbf{x}_{i}, \boldsymbol{\theta}\right)}{\partial \boldsymbol{\theta}}\right|_{\theta}\right)^{\top} \boldsymbol{\Sigma}_{i}^{-1}\left(\left.\frac{\partial \hat{\mathbf{y}}\left(\mathbf{x}_{i}, \boldsymbol{\theta}\right)}{\partial \boldsymbol{\theta}}\right|_{\theta}\right)\right]-2 \sum_{i=1}^{N}\left[\left(\mathbf{y}_{i}-\hat{\mathbf{y}}\left(\mathbf{x}_{i}, \boldsymbol{\theta}\right)\right)^{\top} \boldsymbol{\Sigma}_{i}^{-1}\left(\left.\frac{\partial^{2} \hat{\mathbf{y}}\left(\mathbf{x}_{i}, \boldsymbol{\theta}\right)}{\partial \boldsymbol{\theta} \partial \boldsymbol{\theta}^{\top}}\right|_{\hat{\theta}}\right)\right] .
\end{aligned}
$$

Based on Equations 18 and 19, the Hessian matrix $\mathrm{H}$ can thus be rewritten as follows:

$$
\mathbf{H}=2 \mathbf{F I M}-2 \sum_{i=1}^{N}\left[\left(\mathbf{y}_{i}-\hat{\mathbf{y}}\left(\mathbf{x}_{i}, \boldsymbol{\theta}\right)\right)^{\top} \boldsymbol{\Sigma}_{i}^{-1}\left(\left.\frac{\partial^{2} \hat{\mathbf{y}}\left(\mathbf{x}_{i}, \boldsymbol{\theta}\right)}{\partial \boldsymbol{\theta} \partial \boldsymbol{\theta}^{\top}}\right|_{\boldsymbol{\theta}}\right)\right] .
$$

The relation between the Hessian $\mathrm{H}$ and the FIM is given in Equation 20, and from this equation it can be seen that they differ by the term:

$$
2 \sum_{i=1}^{N}\left[\left(\mathbf{y}_{i}-\hat{\mathbf{y}}\left(\mathbf{x}_{i}, \boldsymbol{\theta}\right)\right)^{\top} \boldsymbol{\Sigma}_{i}^{-1}\left(\left.\frac{\partial^{2} \hat{\mathbf{y}}\left(\mathbf{x}_{i}, \boldsymbol{\theta}\right)}{\partial \boldsymbol{\theta} \partial \boldsymbol{\theta}^{\top}}\right|_{\boldsymbol{\theta}}\right)\right] .
$$

The term consists of three components: the estimation error $\left(\mathbf{y}_{i}-\hat{\mathbf{y}}\left(\mathbf{x}_{i}, \boldsymbol{\theta}\right)\right)$, the inverse measurement error covariance matrix $\boldsymbol{\Sigma}_{i}^{-1}$, and the second derivatives of $\hat{\mathbf{y}}$ to the parameters, also known as the " curvature". For a model which has successfully been calibrated using the available data, the estimation error should be the random (i.e. normally distributed with mean 0 and $\sigma^{2}$ ) measurement error of each point. Therefore, the second derivative terms tend to cancel out when summed over all points $i[35]$, and thus Equation 11 can be reduced to

$$
J(\boldsymbol{\theta}) \approx J(\boldsymbol{\theta})+(\boldsymbol{\theta}-\boldsymbol{\theta})^{\top} \mathbf{F I M}(\boldsymbol{\theta}-\boldsymbol{\theta}) .
$$

As stated before, this is only true for linear models and when the model fits the data perfectly, i.e. the model structure is correct and the global minimum is found [35]. Marsili-Libelli et al. [35] proposed to use the conceptual 
difference between the Hessian matrix $\mathrm{H}$ and the FIM to detect inaccurate parameter results because the optimisation algorithm will be terminating far from the optimum. This method was successfully applied to two simple ecological models using in silico generated data. However, De Pauw [15] pointed out that also other factors beside the premature convergence might influence the difference between the Hessian matrix $\mathrm{H}$ and the FIM. First, the model could be inadequate. Second, by fixing some parameters at specified values, an unsatisfactory model fit might be obtained (although being the global optimum for the selected parameter subset). Therefore, the difference between the Hessian matrix and the FIM can be used as an indicator for model inadequacies, local minima and/or non-normally distributed residuals [15]. In this paper, both the Hessian matrix $\mathrm{H}$ and the FIM will be used to calculate the confidence regions, since this will allow to determine whether the model is calibrated properly.

\section{Optimal experimental design}

The aim is to perform experiments which lead to accurate parameter estimates with minimum experimental effort. Such informative experiments imply that during parameter estimation a small change in parameter value results in a large difference of the cost function $J$. A clearly defined minimum for $J$, can be achieved by maximising the difference between $J(\theta)$ and $J(\theta)$. Maximising the difference between $J(\theta)$ and $J(\theta)$ can be realised by maximising the second order term in Equation 11. In most cases, the second order term is approximated by using the FIM, since this is easier to calculate and reduces computational expenses. In order to maximise the magnitude of the FIM by using an optimisation algorithm, the FIM needs to be reduced to a scalar metric. Various real-valued functions are suggested as metrics and are shown in Table 1. The D-criterion is most commonly used and will also be used in this paper. By maximising the determinant of the FIM the overall volume of the confidence region is reduced. D-optimal experiments possess the attractive property, as opposed to the other design criteria, of being invariant with respect to any rescaling of the parameter units. Although the 
value of the criterion changes as function of the parameter units, the optimal experiment remains the same [무].

Mathematically the optimal experiment, using the $\mathrm{D}$-criterion, is given by Equation 23.

$$
\mathbf{x}_{\mathrm{D}}=\arg \left\{\max _{\mathbf{x} \mathbf{X}}[\operatorname{det}[\mathbf{F I M}(\boldsymbol{\theta}, \mathbf{x})]]\right\}
$$

where $X$ represents the experimental design space and $x_{D}$ the optimal experiment for a specific parameter set $\theta$ using the D-criterion. The optimal experiment is thus only optimal for the parameter set it was designed for. Since the FIM is calculated from the local sensitivity functions (see Equation 18), for nonlinear models the FIM is directly influenced by the parameter values themselves. Therefore, all designs based on the FIM properties are called local designs [41]. Prior to a model calibration no detailed knowledge is available about the parameter values, but this knowledge is important since it will determine the effectiveness of the experimental design. To overcome this problem, more robust Model-based Optimal Experimental Design (rMbOED) methods have been proposed in literature which are insensitive (or at least less sensitive) to the starting values of the different parameters [41, 2]. The use of rMbOED is therefore more suitable for parameter estimation, and will be discussed in the following section.

\section{Robust Optimal Experimental Design}

The aim of robust OED is to design experiments which are suitable for an entire parameter space $\Theta$ and not just for one parameter set $\boldsymbol{\theta}$. Different rMbOED methodologies exist in literature [41, 2]. In this paper the focus will be on the implementation and use of the maximin approach, since it was shown that this approach is superior compared to other robust methodologies [2].

\section{Maximin approach}


The maximin approach, also known as the worst-case approach, aims to optimise the experiment design for any $\boldsymbol{\theta} \in \boldsymbol{\Theta}$ [2]. By searching for the experimental design $\mathbf{x}$ which maximises the information for the worst performing parameter set, this approach tries to find an acceptable performance for all parameter sets $\boldsymbol{\theta}$ in the parameter design space $\boldsymbol{\Theta}$. This results in a robust design $\mathbf{x}_{\mathrm{MMD}}$, which is given by Equation 24 .

$$
\mathbf{x}_{\mathrm{MMD}}=\arg \left\{\max _{\mathbf{x} \mathbf{X}}\left[\min _{\boldsymbol{\theta} \boldsymbol{\theta}}[\operatorname{det}(\mathbf{F I M}[\boldsymbol{\theta}, \mathbf{x}])]\right]\right\}
$$

Asprey and Macchietto [2] stated that Equation 24 can also be written as an infinite dimensional problem, as the constraints must be satisfied for allvalues of $\boldsymbol{\theta}$ within the infinite parameter space $\Theta$ (Equation 25).

$$
\begin{aligned}
& \mathbf{x}_{\mathrm{MMD}}=\arg \left\{\max _{\mathbf{x \in X}, \theta \boldsymbol{\theta}} \Psi\right\} \\
& \text { s.t. } \Psi \leq \operatorname{det}(\mathbf{F I M}[\boldsymbol{\theta}, \mathbf{x}]), \forall \boldsymbol{\theta} \in \boldsymbol{\Theta}
\end{aligned}
$$

Equation 25 represents an infinite dimensional problem, as the constraint must be satisfied for al/values of $\boldsymbol{\theta}$ within the infinite set $\boldsymbol{\Theta}$ [2]. Gustafson [25] suggested a general algorithm to solve such problems for constrained nonlinear optimisation under uncertainty. Instead of calculating the nested optimisation directly, the general algorithm allows to solve the optimisation in two separate steps: First, the experimental design $\mathbf{x}$ is optimised for the worst performing parameter set in a list of parameter sets with unacceptably low performance. Initially, this list only contains the initial parameter guess, but gradually expands during the iterations. Second, for the current design $\mathbf{x}$ the worst performing parameter set $\boldsymbol{\theta}$ within the parameter space $\Theta$ is searched for. If a new parameter set is found which performs worse for the current optimal design, the parameter set is added to the list of parameter sets with low performance and the procedure is repeated. Otherwise it can be concluded that the current optimal design is robust for the parameter space $\boldsymbol{\Theta}$ [2]. The practical implementation of the algorithm is discussed in more detail by Asprey and Macchietto [2]. The maximin optimisation is performed using the particle swarm optimisation (PSO) algorithm, which is a global optimisation method. 


\section{Materials and methods}

\section{Experimental Approach}

Stock solutions

For performing the experiments, different stock solutions were prepared. First, a $20 \mathrm{mM} \mathrm{KH}_{2} \mathrm{PO}_{4} / \mathrm{K}_{2} \mathrm{HPO} 4$ buffer was prepared at $\mathrm{pH}$ 8. From this buffer solution, a stock solution containing $0.1 \mathrm{mM}$ pyridoxal-5'-phosphate (PLP) was prepared which was used to prepare the $500 \mathrm{mM}$ ACE and $40 \mathrm{mM}$ MPPA stock solutions. For all the stock solutions, the $\mathrm{pH}$ was measured and adjusted to obtain the required $\mathrm{pH} 8$. All stock solutions were contained in the fridge to avoid concentration losses due to evaporation. The enzyme solution was prepared daily, using the buffer stock solution without PLP. The enzyme solution was prepared using $\omega$-transaminase (ATA-wt) crude enzyme powder and freeze-dried cells with a specific activity of $1.56 \mathrm{U} / \mathrm{mg}$ and was provided by c-LEcta $\mathrm{GmbH}$, Leipzig, Germany.

\section{Experiments}

The proper amount of buffer with PLP, ACE and MPPA were injected in a 4.5 $\mathrm{mL}$ glass vial. These vials were sealed using a lid with a septum and placed in a thermoshaker which was operated at an orbital agitation of $400 \mathrm{rpm}$ and at a temperature of $30^{\circ} \mathrm{C}$. The sealing was performed to minimise the losses of substrate and products. After heating the solution for about $20 \mathrm{~min}$, the reaction was started by injecting the enzyme solution. The injection time of the enzyme was considered as time zero, and samples were taken after 0.5, 15, 30, 45 and 60 min. Using a manual high-performance liquid chromatography (HPLC) syringe of $25 \mu \mathrm{L}, 20 \mu \mathrm{L}$ samples were taken from the vials and injected in a small HPLC vial containing $180 \mu \mathrm{L}$ of $1 \mathrm{M} \mathrm{NaOH}$.

\section{HPLC}

The samples were analysed ex situ with a reversed-phase chromatography on an Ultimate 3000 HPLC (Dionex, Sunnyvale, CA, USA) equipped with a UV detector. The column was a Gemini ${ }^{\circledR} 3 \mu \mathrm{m}$ NX-C18 $110 \AA$, $100 \times 2.0$ mm (Phenomenex, Torrance, CA, USA), and the analytes were eluted at a flow 
rate of $0.450 \mathrm{~mL} / \mathrm{min}$ in isocratic mode using a mobile phase composition of $65 \%$ Milli-Q aqueous phase $\mathrm{pH} 11$ (adjusted with $\mathrm{NaOH}$ ) and 35\% acetonitrile.

\section{Software}

Python was used to implement the maximin algorithm described by Asprey and Macchietto [2]. The algorithm was implemented in the framework of the pyIDEAS package [47]. Data analysis was performed by using pandas (data

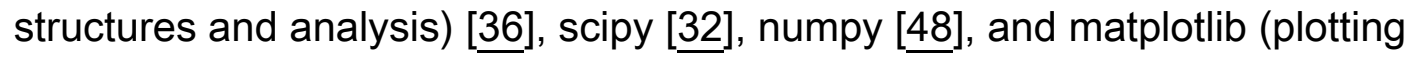
library) [30]. Symbolic derivations were performed using Sympy [46]. Global optimisation problems were tackled by using the Particle Swarm Optimisation (PSO) algorithm of the inspyred package [23]. The calculation of the relative curvature measures [5] was also implemented using numpy, and is discussed in more detail in Appendix A.

\section{Results \& Disussion}

First, the model parameters of Equation $5\left(V_{\mathrm{r}}, K_{\mathrm{ACE}}\right.$, and $\left.K_{\mathrm{MPPA}}\right)$ are estimated using the iterative rMbOED approach. Next, the estimation of the $95 \%$ confidence region is compared with the more accurate likelihood method. Finally, the rMbOED strategy is compared with a straightforward uniform design strategy to illustrate the merit of using rMbOED.

\section{Parameter estimation strategy using iterative rMbOED}

To retrieve the intrinsic parameter values of the backward initial reaction rate, experiments need to be performed at conditions which are most informative. During the rMbOED approach, it is assumed that the model structure is perfect (i.e. valid under all experimental conditions). Therefore, the concentrations of $A C E$ and MPPA are gradually expanded during the different iterations in order to avoid inhibition. Moreover, such an iterative approach is typically more powerful compared to a traditional approach [6].

\section{Preliminary expert-based experiments}


Before starting the actual rMbOED, some preliminary experiments needed to be performed. By gathering such preliminary data, a very rough estimation of the different parameter values and uncertainties can be made. Initially, the experimental design ranges of [ACE] and [MPPA] were limited between 25 and $100 \mathrm{mM}$ and 2.5 and $10 \mathrm{mM}$ respectively. These small concentration ranges were considered to avoid inhibition, and were based on expert knowledge. The preliminary experiments are given in Table 2 , and were used to perform a preliminary parameter estimation of the backward initial rate (Equation 5). The corresponding parameter estimates and uncertainties are provided in Table 2. Since the 95\% confidence intervals are much larger compared to the corresponding estimated parameter values, it is obvious that more informative experiments need to be conducted. To design new experiments, a parameter design space $\boldsymbol{\Theta}$ has to be defined. The parameters $95 \%$ confidence region is used as the parameter design space $\boldsymbol{\Theta}$ for each iteration with a minimum parameter value of zero. For example, the parameter design range of $K_{\mathrm{ACE}}$ for designing iteration 1 is set to [0 mM, $1061 \mathrm{mM}$ ]. This allows to propose experiments which are suitable for all parameter sets located within the $95 \%$ confidence region. Since the size of this confidence region will gradually decrease, the design will become more and more specific during the iterative rMbOED procedure.

The rMbOED approach is now used to design five new experiments with two repetitions for iteration 1 , yielding a total of 10 experiments. All newly designed experiments seem to be located at a concentration of $100 \mathrm{mM}$ of $A C E$, indicating that more information can potentially be found at higher $A C E$ concentrations. As shown in Table 2, the preliminary estimate of $K_{\mathrm{ACE}}$ is 263 $\mathrm{mM}$ which indicates that experiments need to be performed at higher $A C E$ concentrations and is confirmed by Figure 3 . Since the parameter relative sensitivities are used in Figure 3, the sensitivity of the different parameters can be directly compared, yielding that $V_{\mathrm{r}}$ is the most sensitive parameter, followed by $K_{\text {MPPA }}$ and $K_{\text {ACE. }}$. It is interesting that the region where the parameters are most sensitive are quite different, which allows to reduce correlation between the parameters. The sign of the local parameter relative 
sensitivity of $V_{\mathrm{r}}$ is positive, which indicates that an increase of $V_{\mathrm{r}}$ will lead to an increase of the $v_{\text {back }}$ and vice versa. For the other two parameters (KMPPA and $K_{\mathrm{ACE}}$, the sign is negative, indicating that an increase in parameter value will lead to a decrease of $v_{\text {back }}$.

\section{Iteration 1 of $r M b O E D$}

Since no inhibition was observed in the preliminary experiments, the maximum allowed concentrations of [ACE and [MPPA] were doubled to 200 and $20 \mathrm{mM}$ respectively. It is expected that the extended range will yield more information for the parameter calibration, since the parameters are more sensitive at higher concentrations. The newly designed experiments are given in Table 2: iteration 1. For the current and the following iterations, the experiments proposed by the rMbOED algorithm were rounded to concentrations and volumes which were easy to handle in practice. Performing these experiments, 10 additional data points were generated, yielding a total of 20 data points ( 10 from iteration 0 and 10 from iteration 1) which were used to calibrate Equation 5. Compared to the preliminary calibration (iteration 0 ) and iteration 1 , some changes were noticed. First, the $K_{\text {MPPA }}$ value decreased from $21 \mathrm{mM}$ to $3.4 \mathrm{mM}$ and $V_{\mathrm{r}}$ decreased from 34 $\mathrm{nmol} /($ Umin) to $24 \mathrm{nmol} /($ Umin) .

Moreover, the standard deviations (SDs) of all parameters dropped with at least a factor 5 , indicating that the experiments in iteration 1 were more informative. A similar trend was observed for the $95 \%$ confidence intervals (Cls), leading to the fact that for all parameters 0 was no longer within the $95 \% \mathrm{Cl}$. This might seem unimportant, but as long as 0 is part of the $95 \% \mathrm{Cl}$, the parameter has no significant added value for the model and thus may as well be omitted. In order to evaluate the effectivity of the rMbOED approach, the standard deviations between the different iterations need to be compared. The 95\% Cls are dependent on the number of experiments (see Equation 17), and thus make a fair comparison between the different iterations impossible as the $95 \% \mathrm{Cl}$ decreases as the number of experiments increases. 
Since no inhibition was observed in the previous iteration, the maximum allowed concentration of [ACE] was further increased to $300 \mathrm{mM}$. Since $K_{\text {MPPA }}$ is low $(3.4 \pm 3.3 \mathrm{mM})$, it was decided to reduce the maximum [MPPA] concentration to $16 \mathrm{mM}$. The stepwise increase/decrease of the maximum concentrations can look inefficient, however this approach has some advantages: The product concentrations in the current experimental setups are always well below $10 \mathrm{mM}$, so the current range is already high compared to the experiments. Moreover, the model structure is limited to only three parameters instead of requiring two additional parameters to describe the inhibition. More complex models are harder to calibrate and also require more data to estimate the parameters in a reliable way. The estimated parameter values remained fairly constant (small decreases for all parameter values), but the $95 \%$ uncertainty was decreased with more than $30 \%$ for all parameters.

\section{Iteration 3 of $\mathrm{rMbOED}$}

Based on the parameter values of iteration 2, the maximum concentration of [MPPA] was reduced to $10 \mathrm{mM}$ since the estimated $K_{\text {MPPA }}$ value was around $2.7 \mathrm{mM}$ in the previous iteration, and thus will be most sensitive at values below $10 \mathrm{mM}$. The experiments proposed by the rMbOED algorithm were mainly located at the maximum concentration for both ACE and MPPA. Therefore, three out of five newly designed experiments are located at an ACE concentration of $250 \mathrm{mM}$ and a MPPA concentration of $10 \mathrm{mM}$. The newly gathered data allowed to perform a third calibration and uncertainty calculation. Like in previous iterations, the uncertainties for the different parameters further decreased, and it was decided that given the experimental uncertainty, the parameter values and confidence intervals were now sufficiently accurate. The local parameter relative sensitivities for the final iteration are given in Figure 4. Compared to the preliminary iteration (Figure 3), the sensitivities have changed (most pronounced for $K_{\mathrm{MPPA}}$ ), stressing the importance of the parameter value on the local sensitivity and hence on the experimental design. From this figure, it also becomes clear why most of the experiments are designed at the maximum concentration for both 
ACE and MPPA. $V_{\mathrm{r}}$ is the most sensitive parameter, and thus will have the largest impact on the design. Moreover, the two Michaelis constants also show a relatively high sensitivity in this region. These observations indicate that this region is of interest for all kinetic parameters, and thus will play an important role in maximising the D-criterion.

Using the estimated parameter values of both the backward and forward (Appendix B) initial reaction rates, the remaining parameters (i.e. $K_{\text {ilPA, }} K_{\text {iMPPA, }}$ and $K_{\text {eq) }}$ can be estimated (see Appendix B).

\section{Parameter correlation}

The parameter correlations for the different iterations can be calculated based on Equation 16 and are listed in Table 3. The correlation between the different parameters is high, especially between parameters $V_{\mathrm{r}}$ and $K_{\text {ACE }}$. From the local parameter relative sensitivities in Figure 4, it can be seen that the absolute value of the local parameter relative sensitivities increases when increasing both $[A C E$ and $[M P P A]$, making it difficult to decouple the effect of both parameters. The parameter correlations can be reduced by increasing the maximum $A C E$ concentration to about $500 \mathrm{mM}$, since the $K_{\mathrm{ACE}}$ will show a decreasing sensitivity at higher concentrations (not shown).

\section{Measurement uncertainty of backward initial rate}

For the forward reaction rate a measurement uncertainty analysis had already been carried out. This measurement uncertainty analysis yielded that the measurement uncertainty relative to the reaction rate, was following a normal distribution ( $\mu=0$ and $\sigma\left(v_{\text {forw }}\right)=0.10 v_{\text {forw }}$ ).

Therefore, for the backward reaction rate the same measurement uncertainty was assumed and used to perform the rMbOED, since the same equipment and solutions were used. It would be possible to estimate the uncertainty during the actual experimentation, but this would require at least three repetitions for each experiment, which would increase the experimental effort considerably. Otherwise, estimating the uncertainty from the offset between the data and model is only reasonable when a sufficiently high number of 
experimental data points have been collected, and thus is preferably done after the data collection. However, now it is verified whether the use of the relative error of the forward initial reaction rate is also valid for the backward initial reaction rate. The relative error $\epsilon_{\mathrm{rel}, i}$ is given in Equation 26 and the corresponding histogram for the gathered data and calibrated model is given in Figure 5.

$$
\epsilon_{\mathrm{rel}, i}=\frac{\mathbf{y}_{i}-\hat{\mathbf{y}}_{i}(\boldsymbol{\theta}, \mathbf{x})}{\mathbf{y}_{i}}
$$

The null hypothesis was that the relative measurement uncertainty $\left(\epsilon_{\mathrm{rel}, i}\right)$ was indeed following a normal distribution. This null hypothesis was tested using an omnibus test of normality proposed by D'Agostino [12], and is appropriate to detect deviations from normality due to either skewness or kurtosis [13]. This normality test is available in the scipy package (scipy.stats.normaltest) [32], and returns a two-sided $\chi^{2}$ probability for the hypothesis test. The test yielded a p-value of 0.595 , and thus the null hypothesis could not be rejected. Therefore it was concluded that the relative measurement uncertainty was following a normal distribution with $\sigma\left(v_{\text {back }}\right)=0.104 v_{\text {back }}$. The approach of estimating the measurement error by evaluating the difference between the model prediction and measurements is only valid if the model in Equation 5 represents the true model. The measurements of Table 2 and the model prediction using the parameter values and covariance matrix obtained in the third iteration are shown in Figure 6. From this Figure, it can be observed that the measurement uncertainty (which is calculated by using Equation 26, and thus only provides an estimate of the real error) is much larger compared to the predicted $95 \%$ model confidence interval. The 95\% model confidence interval is calculated by propagating the uncertainties on the parameter estimates by using the covariance matrix $\boldsymbol{\Phi}$ [39]. It can also be noticed that the measurements performed in iteration 3 are consistently overpredicted by the model. The enzyme used for this iteration was from a different batch, probably increasing the overall uncertainty of the parameter estimates. However, in this way the potential deviations between the batches originating 
from the enzyme production are also incorporated in the parameter uncertainty.

\section{Difference between FIM and Hessian matrix $\mathrm{H}$}

In the previous calculations, the $\mathbf{F I M}^{-1}$ was used to estimate the covariance matrix. However, the FIM is only a good estimate of the Hessian $\mathrm{H}$ if no important offset exists between the model and the data (Equation 21). Since the model under study is an algebraic model, it is straightforward to calculate the second derivatives of $\hat{\mathbf{y}}$ to the parameters. The $\mathbf{H}-2 \mathbf{F I M}$ is given in Equation 27 and is very small compared to the $\mathrm{H}$, since the maximum relative deviation between the different terms (i.e. $\left.\left(\mathbf{H}_{i j}-2 \mathbf{F I M}_{i j}\right) / \mathbf{H}_{i j}\right)$ ) is smaller than $2 \%$ ). Therefore based on Marsili-Libelli et al. [35], it is concluded that the model is calibrated properly.

$$
\mathbf{H}-2 \mathbf{F I M}=\left[\begin{array}{ccc}
{[c r r] 0.00 e+00} & -4.66 e-04 & -1.05 e-05 \\
-4.66 e-04 & 2.45 e-01 & -3.56 e-03 \\
-1.05 e-05 & -3.56 e-03 & 3.00 e-05
\end{array}\right]
$$

\section{Importance of curvature for parameter confidence estimation}

Using the procedure proposed by Bates and Watts [5] (A), the importance of the curvature of the objective function $J$ can be assessed. It is found that the relative intrinsic curvature $c^{\prime}$ is equal to 0.034 and the relative parametereffect curvature $c^{\theta}$ is equal to 0.296 . From these results, it can already be concluded that the intrinsic curvature is much less important compared to the parameter-effects curvature, which is in accordance with previous observations of Bates and Watts [4] and Donaldson and Schnabel [18]. In order to provide a sufficiently low deviation from the tangent plane at a distance $\sqrt{F}$ from the tangent point, $c \sqrt{F}$ needs to be (much) smaller than 1 , where $F$ represents the value of the $F$-distribution. The square root of the critical $F$-value $(F(N, N-P, 0.95))$ here found is equal to 1.70. Bates and Watts [5] stated that $c \sqrt{F}$ should be lower than 0.3 , to have deviations lower than $15 \%$. Since $c^{\theta} \sqrt{F}$ is equal to 0.503 , it is expected that the parametereffects curvature is important and thus the FIM and $\mathrm{H}$ will not provide proper estimates of the parameter confidence intervals. To determine how close the 
current confidence region prediction is compared to reality, independent samples were taken to estimate the likelihood confidence region (Equation 9). The likelihood method is suitable to estimate the confidence region, since the intrinsic curvature is very low and thus will provide a good approximation of the confidence regions. 1000000 random parameter samples are taken from uniform distributions, for which the ranges are given in Table 4 .

Only 14876 samples were found to be located within the $95 \%$ likelihood confidence region. The approximated confidence regions for the different methods are shown in Figure 7. From this Figure, it is clear that the use of the FIM and Hessian matrix $\mathrm{H}$ to construct the confidence ellipses, yield the same result. However, these linear approximations differ considerably from the likelihood confidence region, and thus it can be concluded that it is important to determine the curvature of the objective function, in order to assess whether the linear approximation methods yield reliable results. In this case, the confidence intervals are overpredicted for low parameter values, and underpredicted for high parameter values. These asymmetric confidence intervals are typical for nonlinear models, since only linear models will yield a symmetric, ellipsoidal confidence region [18].

\section{In silico uniform design approach}

To illustrate the added value of the iterative rMbOED strategy, the results are compared with an in silico traditional uniform design based on the initial ranges of the independent variables ([ACE and [MPPA]). For the rMbOED, 20 experiments were conducted with two repetitions for each experiment. To allow comparison, the same number of experiments is used for the in silico uniform design, using the estimated parameter values of iteration 3 of the rMbOED. Both design strategies are shown in Figure 8, and as expected the design space for the uniform design is much smaller since it uses the initial product ranges.

Assuming that the calibrated parameter values and the measurement error calculated earlier $\left(\sigma\left(v_{\text {back }}\right)=0.104 v_{\text {back }}\right)$ are correct, the parameter confidence levels for the uniform design of Figure 8 can be calculated. The $95 \%$ 
confidence intervals $(\mathrm{Cl})$ for the uniform design strategy are given in Table 5 . Compared to the iterative rMbOED strategy, the information content is much lower for the uniform design strategy since the $\operatorname{det}[\mathbf{F I M}]$ is about ten times lower. This also results in confidence intervals which are two times larger for the same experimental effort. This shows that by using the iterative rMbOED strategy, experimental effort can be reduced by indicating and only performing experiments of interest. This is especially true when the a priori parameter information is low and/or the model is highly nonlinear. In these cases, it is impossible to come up with an experimental design which is informative and efficient. However, an iterative procedure allows to update the parameter values and uncertainties during the experimentation, and allows to identify and narrow the experimental regions of interest. In Figure 8, only a uniform design for the initial design space is considered, which might seem unfair to be used for a comparison. However, initially only a limited amount of information is available about the design space, and thus in practice this small design space would be used to design the experiments. If the (a priori unknown) maximum design space of the rMbOED would be used for the uniform design, the parameter uncertainties would be close to those of the rMbOED, but still be higher (about $2 \%$ ). This comparison is also not completely fair, since the iterative rMbOED only gradually expands from the initial to the maximum design space. However, this small difference raises the question to what extent an optimal experimental design approach will outweigh an intuitive planning based on the experimental interpretation of the results. Bauer et al. [6] performed such a comparison, and found that the standard deviations of an intuitive design by an experienced experimentalist were about $30 \%$ higher compared to the model-based design. This illustrates that the use of MbOED is generally superior, and yields more accurate parameter estimation results.

\section{Conclusions}

The iterative robust model-based optimal experimental design (rMbOED) strategy was applied to the backward initial reaction rate (i.e. a submodel of the plain ping-pong bi-bi model), and proved to be powerful and superior to 
the uniform design strategy. By using rMbOED, experimental efforts can be reduced and the experimental region of interest can be identified. During the iterative $\mathrm{rMbOED}$, the experimental design space $\mathbf{X}$ was gradually expanded to include informative design regions. Since no detailed information was available prior to the experimentation a suitable experimental design range was unknown, and thus a conservative design space was considered initially. Using data collected earlier, the remaining model parameters were successfully calibrated, and the full model in Equation 2 was validated for short term experiments $(<7 \mathrm{~h})$. However, large deviations between the data and model predictions were found at long reaction times. This is probably related to enzyme instability and/or precipitation.

The use of linearisation techniques like the Fisher Information Matrix (FIM) to approximate the confidence region of the parameters, was found to deviate significantly from the likelihood confidence region. It was found that the confidence region predicted by the FIM overpredicted the lower $95 \%$ confidence interval boundary, but underpredicted the upper 95\% confidence interval boundary. The relative curvature measures proposed by Bates and Watts [5], allowed to determine whether the FIM is appropriate to approximate the confidence region, and thus should be always calculated when using the FIM for nonlinear models.

\section{Acknowledgements}

Financial support by the European Union FP7 Project BIOINTENSE Mastering Bioprocess integration and intensification across scales (Grant Agreement Number 312148) is gratefully acknowledged.

\section{A Curvature measures of nonlinearity}

The second and higher order derivates of a linear function with respect to the parameters are zero. Therefore, Bates and Watts [5] used second order derivatives of the expectation function to measure the nonlinearity of a model. They introduced a $N \times P$ derivative matrix $\dot{\mathbf{V}}$, where each row contains the gradient of $\hat{\mathbf{y}}$ at a specific sample point $\mathbf{x}_{i}$ to the parameters $\boldsymbol{\theta}$ 
$\dot{\mathbf{V}}=\frac{\partial \hat{\mathbf{y}}(\mathbf{x}, \boldsymbol{\theta})}{\partial \boldsymbol{\theta}}$

and a $N \times P \times P$ second order derivate matrix $\ddot{\mathbf{V}}$

$\ddot{\mathbf{V}}=\frac{\partial^{2} \hat{\mathbf{y}}(\mathbf{x}, \boldsymbol{\theta})}{\partial \boldsymbol{\theta} \partial \boldsymbol{\theta}^{\top}}$

where each face of $\ddot{\mathrm{V}}_{i}$ of $\ddot{\mathrm{V}}$ is a complete $P \times P$ second derivative matrix, or Hessian. From $\ddot{\mathbf{V}}$ the $P(P+1) / 2$ nonredundant acceleration vectors can be easily extracted to construct a matrix $\ddot{\mathbf{W}}$ and be combined with the tangent vectors in $\dot{\mathbf{V}}$ to give

$\mathbf{D}=(\dot{\mathbf{V}}, \ddot{\mathbf{W}})$.

By performing a $Q R$ decomposition on $\mathrm{D}$, the different acceleration vectors are projected into the tangent plane and into the space normal to the tangent plane but spanned by the acceleration vectors.

$\mathbf{D}=\mathbf{Q R}=\left[\mathbf{Q}_{1}\left|\mathbf{Q}_{1}^{\top}\right| \mathbf{Q}_{2}\right]\left[\begin{array}{cc}\mathbf{R}_{11} & \mathbf{A}^{\theta} \\ \mathbf{0} & \mathbf{A}^{i} \\ \mathbf{0} & \mathbf{0}\end{array}\right]$

where $\mathbf{R}_{11}$ is a $P \times P$ upper-left triangular matrix, which equals the full $R$ matrix when performing a $Q R$ decomposition only on $\dot{\mathbf{V}}$. The matrices $\mathbf{A}^{\theta}$ and $\mathbf{A}^{i}$ have dimensions $P \times P(P+1) / 2$ and $P^{\prime} \times P(P+1) / 2$ respectively, and are used to form the parameter effects and intrinsic components of the curvature or acceleration array $\ddot{\mathbf{A}}$. The tangential components of the acceleration vectors are contained in $\mathbf{A}^{\theta}$, while the normal components are contained in $\mathbf{A}^{i}$. The extent to which the acceleration vectors lie outside the tangent plane provides a measure of how much the expectation surface deviates from a plane, which is called the intrinsic nonlinearity since it does not depend on the parametrisation chosen for the expectation function, but only on the experimental design and the expression of the expectation function. However, the projections of the acceleration vectors in the tangential plane depend on the parametrisation of the model, and measure the 
nonuniformity of the parameter lines on the tangent plane, which is called the parameter effects nonlinearity. [5]

Bates and Watts [5] pointed out that the curvatures, are measured in units of $1 /$ response, and thus the values depend on the scaling of the data. To remove this dependence, a $\left(P+P^{\prime}\right) \times P \times P$ relative curvature array can be calculated (Equation 32).

$$
\mathbf{C}=\mathbf{R}_{11}^{-\top} \ddot{\mathbf{A}} \mathbf{R}_{11}^{-1} s \sqrt{P}
$$

where $s$ represents $\sqrt{S S E /(N-P)}$. For data analysis, a simple overall measure of the nonlinearity is needed to assess the quality of a linear approximation. Bates and Watts [5] proposed a simple overall scalar measure, i.e. root mean square (RMS) curvature measure, which is the square root of the average over all directions of the squared curvature [8], calculated by

$$
c^{2}=\frac{1}{P(P+2)} \sum_{n}\left[2 \sum_{p=1}^{P} \sum_{q=1}^{P} C_{n p q}^{2}+\left(\sum_{p=1}^{P} C_{n p p}\right)^{2}\right] .
$$

where index $n$ goes from 1 to $P$ for $c^{\theta}$ and from $P+1$ to $P+P^{\prime}$ for $C$.

\section{B Parameter estimation remaining model parts}

The data for the forward initial reaction rate and the dissociation parameters were gathered separately, and thus the presented rMbOED methodology was not applied for the model calibration of the remaining model parts. All parameter estimates, uncertainties and correlation derived from the FIM are given in Tables 6 and 7.

\section{B.1 Forward initial reaction rate}

The forward initial reaction rate in Equation 4 contains three parameters, i.e. $V$ f, $K_{\mathrm{IPA}}$, and $K_{\mathrm{BA}}$. In order to estimate the parameter values 54 experiments were conducted, for which the substrate concentrations were varied between 20 and $800 \mathrm{mM}$ for IPA and between 0.5 and $10 \mathrm{mM}$ for $B A$. The initial 
product concentrations for $[M P P A]$ and $[A C E]$ were equal to $0 \mathrm{mM}$. After the data collection, the model was calibrated using two different error distributions, i.e. the absolute constant error and the relative error. It was found that the absolute constant error distribution was no suitable error distribution ( $p$-value of 0.00715 ), and thus was rejected. The relative error distribution seemed to represent the error distribution well, for $\mu=0$ and $\sigma=0.099 v$. The calibrated parameter values and $95 \% \mathrm{Cls}$ are given in Table 6 . The gathered forward initial reaction rate data and calibrated model are shown in Figure 9.

From Figure 9 it is clear that the model is able to predict the data well. However, at high concentrations of IPA, the measurement uncertainty is higher. This is probably related to the operational instability of the enzyme at high amine donor-to-acceptor ratios.

\section{B.2 Dissociation parameters}

After estimating the parameters of both the forward and backward initial reaction rate, three parameters remained to be calibrated: $K_{\text {eq, }} K_{\text {ilPA, }}$ and $K_{\text {iMPPA. }}$ Using the Haldane relationship from Equation $3, K_{\text {eq }}$ could already be estimated. To estimate the other two parameters, additional initial rate experiments were performed. The substrate concentrations for [IPA] and $[B A]$ were fixed to 450 and $10 \mathrm{mM}$ respectively. The product concentration of [ACE] was varied between 0 and $100 \mathrm{mM}$ and that of [MPPA] was varied between 0 and $20 \mathrm{mM}$, and is shown in Figure 10. Twelve experiments were carried out, allowing to calibrate the two remaining parameters (Table 6).

\section{B.3 Model validation}

A model validation was performed at different initial conditions and it was found that the model prediction showed a good correspondence with the experiments at low reaction times ( $<7 \mathrm{~h}$ ). At higher reaction times (up to $17 \mathrm{~h}$ ), $[M P P A]$ was overpredicted and $[B A]$ underpredicted. Two possible reasons can be formulated: First, the model in Equation 2 might be unsuitable for predicting the concentrations for high reaction times. Second, the enzyme 
was suffering from some stability issues and precipitation was observed, possibly influencing the reaction kinetics. As the model can be regarded as calibrated and validated for low reaction times, the final parameter values, $95 \%$ confidence intervals and correlation are given in Table 6 . The 95\% confidence intervals and correlations were calculated using the FIM (Table 7), and since the model in Equation 2 is nonlinear, this is only a rough approximation (see section).

\section{Literature Cited}

[1] Naweed Al-Haque, Paloma A Santacoloma, Watson Neto, Pär Tufvesson, Rafiqul Gani, and John M Woodley. A robust methodology for kinetic model parameter estimation for biocatalytic reactions. Biotechnol Prog, 28(5):11861196, 2012.

[2] S.P. Asprey and S. Macchietto. Designing robust optimal dynamic experiments. J Process Contr, 12(4):545-556, 2002.

[3] Filipe Ataíde and Bernd Hitzmann. When is optimal experimental design advantageous for the analysis of Michaelis-Menten kinetics? Chemometrics and Intelligent Laboratory Systems, 99(1):9-18, 2009.

[4] Douglas M. Bates and Donald G Watts. Relative Curvature Measures of Nonlinearity. J Roy Stat Soc B Met, 42(1):1-25, 1980.

[5] Douglas M. Bates and Donald G. Watts. Curvature Measures of Nonlinearity. In Nonlinear Regression Analysis and Its Applications, Wiley Series in Probability and Statistics, pages 232-263. John Wiley \& Sons, Inc., Hoboken, NJ, USA, 1988.

[6] Irene Bauer, Hans Georg Bock, Stefan Körkel, and Johannes P Schlöder. Numerical methods for optimum experimental design in DAE systems. $J$ Comput Appl Math, 120(1-2):1-25, 2000.

[7] E M L Beale. Confidence Regions in Non-Linear Estimation. J Roy Stat Soc B Met, 22(1):41-88, 1960. 
[8] Lamia Benabbas, Steven P. Asprey, and Sandro Macchietto. CurvatureBased Methods for Designing Optimally Informative Experiments in Multiresponse Nonlinear Dynamic Situations. Ind Eng Chem Res, 44(18):7120-7131, 2005.

[9] Roland Brun, Peter Reichert, and Hans R Kfinsch. Practical identifiability analysis of large environmental simulation. Water Resour Res, 37(4):10151030, 2001.

[10] Bing H. Chen, Edward G. Hibbert, Paul A. Dalby, and John M. Woodley. A new approach to bioconversion reaction kinetic parameter identification. A/ChE J, 54(8):2155-2163, 2008.

[11] Athel Cornish-Bowden. Fundamentals of Enzyme Kinetics. Portland Press Ltd., London, 3 edition, 2004.

[12] R. B. D'Agostino. An omnibus test of normality for moderate and large size samples. Biometrika, 58(2):341-348, 1971.

[13] Ralph D'Agostino and E. S. Pearson. Tests for Departure from Normality. Biometrika, 60(3):613, 1973.

[14] D J W De Pauw, K Steppe, and B De Baets. Identifiability analysis and improvement of a tree water flow and storage model. Math. Biosci., 211(2):314-332, 2008.

[15] Dirk De Pauw. Optimal Experimental Design for calibration of bioprocess models: a validated software toolbox. PhD thesis, Ghent University, Belgium, 2005.

[16] M Dixon. The determination of enzyme inhibitor constants. Biochem J, 55(1):170-1, 1953.

[17] Denis Dochain and Peter Vanrolleghem. Dynamical Modelling and Estimation in Wastewater Treatment Processes. IWA Publishing, London, UK, 2001. 
[18] Janet R. Donaldson and Robert B Schnabel. Computational Experience with Confidence Regions and Confidence Intervals for Nonlinear Least Squares. Technometrics, 29(1):67-82, 1987.

[19] John E. Dowd and Douglas S. Riggs. A Comparison of Estimates of Michaelis-Menten Kinetic Constants from Various Linear Transformations. $J$ Biol Chem, 240(2):863-869, 1965.

[20] G. S. Eadie. The Inhibition of Cholinesterase by Physostigmine and Prostigmine. J Biol Chem, 146(1):85-93, 1942.

[21] R Eisenthal and A Cornish-Bowden. The direct linear plot. A new graphical procedure for estimating enzyme kinetic parameters. Biochem J, 139(3):715-20, 1974.

[22] Valerii V. Fedorov and Peter Hackl. Convex Design Theory. In Mode/Oriented Design of Experiments, Lecture Notes in Statistics, page 125. Springer New York, New York, NY, 1997.

[23] Aaron Garrett. Inspyred: Bio-inspired Algorithms in Python, 2012.

[24] Daniel Goujot, Xuân Meyer, and Francis Courtois. Identification of a rice drying model with an improved sequential optimal design of experiments. $J$ Process Contr, 22(1):95-107, 2012.

[25] Sven-Ake Gustafson. A Three-Phase Algorithm for Semi-Infinite Programs. In Anthony V. Fiacco and Kenneth O. Kortanek, editors, SemiInfinite Programming and Applications, volume 215 of Lecture Notes in Economics and Mathematical Systems, pages 138-157. Springer Berlin Heidelberg, Berlin, Heidelberg, 1983.

[26] K. P. Hadeler, Dragan Jukić, and Kristian Sabo. Least-squares problems for Michaelis-Menten kinetics. Math Method App/ Sci, 30(11): 1231-1241, 2007. 
[27] C S Hanes. Studies on plant amylases: The effect of starch concentration upon the velocity of hydrolysis by the amylase of germinated barley. Biochem J, 26(5):1406-21, 1932.

[28] B. H. J. Hofstee. On the Evaluation of the Constants $\mathrm{Vm}$ and $\mathrm{Km}$ in Enzyme Reactions. Science, 116(3013):329-331, 1952.

[29] Andrea Holmberg. On the practical identifiability of microbial growth models incorporating Michaelis-Menten type nonlinearities. Mathematical Biosciences, 62(1):23-43, 1982.

[30] John D. Hunter. Matplotlib: A 2D Graphics Environment. IEEE Comput Sci Eng, 9(3):90-95, 2007.

[31] Bum-Yeol Hwang, Byung-Kwan Cho, Hyungdon Yun, Kinera Koteshwar, and Byung-Gee Kim. Revisit of aminotransferase in the genomic era and its application to biocatalysis. J Mol Catal B-Enzym, 37 (1-6):47-55, dec 2005.

[32] Eric Jones, Travis Oliphant, Pearu Peterson, and Others. SciPy: Open source scientific tools for Python. http://www.scipy.org/, 2001.

[33] Hans Lineweaver and Dean Burk. The Determination of Enzyme Dissociation Constants. J Am Chem Soc, 56(3):658-666, 1934.

[34] Lennart Ljung. System identification - Theory for the User. Prentice-Hall, 2 edition, 1999.

[35] S. Marsili-Libelli, S. Guerrizio, and N. Checchi. Confidence regions of estimated parameters for ecological systems. Ecol Model, 165(2-3):127-146, jul 2003.

[36] Wes McKinney. Data Structures for Statistical Computing in Python. In Proceedings of the 9th Python in Science Conference, pages 51-56, 2010.

[37] Anton Moser. Bioprocess technology: kinetics and reactors. Springer Science \& Business Media, 2012. 
[38] John A Nelder and Roger Mead. A simplex method for function minimization. Comput J, 7(4):308-313, 1965.

[39] Martin Omlin and Peter Reichert. A comparison of techniques for the estimation of model prediction uncertainty. Ecol Model, 115(1):45-59, 1999.

[40] W.H. Press, S.A. Teukolsky, W.T. Vetterling, and B.P. Flannery. Numerical Recipes in C: the art of scientific computing. Cambridge University Press, Cambridge, UK, 2 edition, 1992.

[41] Luc Pronzato and Eric Walter. Robust experiment design via maximin optimization. Math Biosci, 89(2):161-176, 1988.

[42] Francesco Ranaldi, Paolo Vanni, and Eugenio Giachetti. What students must know about the determination of enzyme kinetic parameters. Biochem Educ, 27(2):87-91, 1999.

[43] G. A. F. Seber and C. J. Wild. Nonlinear Regression. John Wiley \& Sons, Inc., 1989.

[44] Irwin Segel. Enzyme Kinetics: Behavior and Analysis of Rapid Equilibrium and Steady-State Enzyme Systems. John Wiley \& Sons, New York, page 957, 1975.

[45] Jong Shik Shin and Byung Gee Kim. Kinetic modeling of omegatransamination for enzymatic kinetic resolution of alpha-methylbenzylamine. Biotechnol Bioeng, 60(5): 534-40, 1998.

[46] SymPy Development Team. SymPy: Python library for symbolic mathematics, 2014.

[47] Timothy Van Daele, Stijn Van Hoey, and Ingmar Nopens. pyIDEAS: an Open Source Python Package for Model Analysis. In Comput Aided Chem Eng, volume 37, pages 569-574, 2015. 
[48] Stefan van der Walt, S Chris Colbert, and Gael Varoquaux. The NumPy Array: A Structure for Efficient Numerical Computation. Comput. Sci. Eng., 13(2):22-30, 2011.

[49] $P$ Vanrolleghem, M Van Daele, and D Dochain. Practical identifiability of a biokinetic model of activated sludge respiration. Water Res, 29(11):25612570, 1995.

[50] É Walter and L Pronzato. Identification of parametric models from experimental data. Springer-Verlag, Heidelberg, xviii edition, 1997.

Fig. 1 Schematic overview of the iterative optimal experimental design procedure [17].

Fig. 2 Conversion of isopropylamine and benzylacetone to acetone and 1methyl-3-phenylpropylamine by $\omega$-transaminase $(\omega-T A)$ in presence of coenzyme pyridoxal-5'-phosphate (PLP).

Fig. 3 Local parameter relative sensitivity for the different parameters of the backward initial reaction rate for the estimated parameter values of the preliminary iteration $\left(V_{\mathrm{r}}=34 \mathrm{nmol} /(U \mathrm{~min}), K_{\mathrm{MPPA}}=21 \mathrm{mM}\right.$ and $K_{\mathrm{ACE}}=263$ $\mathrm{mM})$. Higher absolute values, i.e. larger deviation from zero, means that the parameter has more influence.

Fig. 4 Local parameter relative sensitivity for the different parameters of the backward initial reaction rate for the estimated parameter values of the third/final iteration $\left(V_{\mathrm{r}}=21.7 \mathrm{nmol} /(\mathrm{Umin}), K_{\mathrm{MPPA}}=2.96 \mathrm{mM}\right.$ and $K_{\mathrm{ACE}}=250$ $\mathrm{mM})$.

Fig. 5 Histogram of the relative error $\epsilon_{\mathrm{rel}, i}$ (Equation 26). The red line represents a normal probability density function with with mean $\mu$ and standard deviation $\sigma$ equal to 0 and 0.104 respectively.

Fig. 6 The model prediction $v_{\text {back }}$ is shown for the different experiments shown in Table 2. The measurement uncertainty depicted is twice the standard 
deviation shown in Figure 5, and thus is only an estimate of the actual measurement error.

Fig. 7 The confidence regions using the linear approximation $\left(\mathrm{CR}_{\mathrm{FIM}}\right.$ and $\left.\mathrm{CR}_{\text {Hessian }}\right)$ show consistent results. Since the parameter-effects curvature is important, the likelihood confidence region $\left(\mathrm{CR}_{\text {Likelihood }}\right)$ yields a more accurate approximation of the actual confidence region.

Fig. 8 The experimental design strategies for the uniform design and robust model-based optimal experimental design ( $\mathrm{rMbOED}$ ). The gray areas show the design space, i.e. the ranges in which an experiment could be designed. The maximum design space is shown for the rMbOED, since it was gradually expanded during the rMbOED iterations.

Fig. 9 The model prediction vforw corresponds well with the experimental data. However, at high IPA concentrations the measurement uncertainty increases.

Fig. 10 The experiments to estimate the dissociation parameters were performed at varying product concentrations ([ACE and $[M P P A])$ and constant substrate concentrations $([I P A]=450 \mathrm{mM}$ and $[B A]=10 \mathrm{mM})$.

Table 1 The different "alphabetic" optimal design criteria based on the FIM properties $[\underline{22}, \underline{50}]$.

\section{Design Name Criterion}

A-optimal $\quad \min \left\{\operatorname{tr}\left(\mathbf{F I M}^{-1}\right)\right\}$

Modified A-optimal $\max \{\operatorname{tr}(\mathbf{F I M})\}$

D-optimal $\quad \max \{\operatorname{det}(\mathbf{F I M})\}$

E-optimal $\quad \max \left\{\lambda_{\min }(\mathbf{F I M})\right\}$

Modified E-optimal $\min \left\{\lambda_{\max }(\mathbf{F I M}) / \lambda_{\min }(\mathbf{F I M})\right\}$

$\operatorname{tr}()$ : sum of eigenvalues; $\operatorname{det}()$ : product of eigenvalues 
Table 2 Experiments conducted for each iterative step of the rMbOED. The parameter estimates and uncertainties are always based on the cumulative dataset, i.e. the parameter estimation of iteration $i+1$ also takes into account the data gathered in iterations $i, i-1, \ldots, 0$.

\section{Ex Condition Measurement}

Iteration

p.

$\mathbf{s}$

$[A C[M P P$

nr. E] $A] \quad v_{\text {rep, } 1}$

$[\mathrm{m} \quad[\mathrm{nmol} /(\mathrm{U}$

M] $\quad[\mathrm{mM}] \mathrm{min})]$
S

$$
v_{\text {rep, } 2}
$$

Estimated parameter

val $95 \%$

ue $\quad \mathrm{Cl} \quad(\mathrm{SD})$

preliminary expert3.4 based design

$\begin{array}{lllll}1 & 50 & 5 & 3.25 & 3\end{array}$

\begin{tabular}{|c|c|c|c|c|c|c|c|}
\hline & & & & 4.5 & $K_{\mathrm{AC}}$ & & 33 \\
\hline \multirow[t]{2}{*}{2} & 75 & 7.5 & 4.79 & 7 & E & $=263 \pm 798$ & $(7)$ \\
\hline & & & & 4.7 & $K_{\mathrm{MP}}$ & & \\
\hline \multirow[t]{2}{*}{3} & 75 & 10 & 5.39 & 6 & PA & $=21 \pm 68$ & $(28)$ \\
\hline & & & & 5.1 & & & \\
\hline \multirow[t]{2}{*}{4} & 100 & 7.5 & 5.23 & 3 & $V_{r}$ & $=34 \pm 86$ & (36) \\
\hline & & & & 6.2 & & & \\
\hline \multirow[t]{2}{*}{5} & 100 & 10 & 6.28 & 8 & & & \\
\hline & & & & 2.7 & & & \\
\hline \multirow[t]{2}{*}{6} & 35 & 10 & 2.93 & 7 & & & \\
\hline & & & & 8.0 & $K_{\mathrm{AC}}$ & & \\
\hline \multirow[t]{2}{*}{7} & 115 & 20 & 8.04 & 9 & E & $=263 \pm 161$ & (76) \\
\hline & & & & 6.8 & $K_{\mathrm{MP}}$ & & \\
\hline \multirow[t]{2}{*}{8} & 200 & 2.5 & 6.86 & 9 & $\mathrm{PA}$ & $=3.4 \pm 3.2$ & (1.5) \\
\hline & & & & 8.2 & & & \\
\hline \multirow[t]{2}{*}{9} & 200 & 7.5 & 8.47 & 1 & $V_{\mathrm{r}}$ & $=24 \pm 12$ & (5.5) \\
\hline & & & & 8.9 & & & \\
\hline 10 & 200 & 15 & 9.86 & 6 & & & \\
\hline
\end{tabular}




\begin{tabular}{|c|c|c|c|c|c|c|c|c|c|}
\hline \multirow[b]{2}{*}{ Iteration } & \multirow{2}{*}{$\begin{array}{l}\text { Ex } \\
\text { p. }\end{array}$} & \multicolumn{2}{|c|}{ Condition } & \multicolumn{2}{|c|}{ Measurement } & \multirow{2}{*}{\multicolumn{4}{|c|}{ Estimated parameter }} \\
\hline & & & $\mathbf{s}$ & & $\mathbf{s}$ & & & & \\
\hline & & & & & 3.7 & & & & \\
\hline \multirow[t]{10}{*}{2} & 11 & 50 & 12 & 3.39 & 3 & & & & \\
\hline & & & & & 4.5 & $K_{\text {AC }}$ & & & \\
\hline & 12 & 105 & 2.5 & 4.61 & 5 & E & $=262$ & $2 \pm 111$ & $(54)$ \\
\hline & & & & & 8.6 & $K_{\mathrm{MP}}$ & & & 0.8 \\
\hline & 13 & 185 & 16 & 8.08 & 2 & PA & $=2.7$ & \pm 1.8 & $\left(\begin{array}{ll}7 & )\end{array}\right.$ \\
\hline & & & & & 10. & & 23. & & \\
\hline & 14 & 250 & 10 & 10.19 & 01 & $V_{\mathrm{r}}$ & $=1$ & \pm 7.6 & $(3.7)$ \\
\hline & & & & & 8.8 & & & & \\
\hline & 15 & 300 & 2.5 & 8.59 & 4 & & & & \\
\hline & & & & & 2.9 & & & & \\
\hline \multirow[t]{9}{*}{3} & 16 & 50 & 10 & 2.74 & 9 & & & & \\
\hline & & & & & 5.5 & $K_{\mathrm{AC}}$ & & & \\
\hline & 17 & 190 & 2.5 & 5.15 & 7 & E & $=250$ & \pm 81 & $(40)$ \\
\hline & & & & & 8.7 & $K_{\mathrm{MP}}$ & 2.9 & & 0.7 \\
\hline & 18 & 250 & 10 & 8.40 & 4 & PA & $=6$ & \pm 1.48 & $(3)$ \\
\hline & & & & & 8.7 & & 21. & & \\
\hline & 19 & 250 & 10 & 9.74 & 2 & $V_{\mathrm{r}}$ & $=7$ & \pm 5.3 & $(2.6)$ \\
\hline & & & & & 8.4 & & & & \\
\hline & 20 & 250 & 10 & 9.11 & 9 & & & & \\
\hline
\end{tabular}

Table 3 Parameter correlations for the different iterations.

Iteration $V_{\mathrm{r}}$ vs $K_{\mathrm{MPPA}} V_{\mathrm{r}}$ vs $K_{\mathrm{AcE}} K_{\mathrm{MPPA}}$ vs $K_{\mathrm{ACE}}$

$\begin{array}{llll}1 & 0.876 & 0.876 & 0.807\end{array}$

$\begin{array}{llll}2 & 0.866 & 0.982 & 0.790\end{array}$

$\begin{array}{llll}3 & 0.860 & 0.973 & 0.790\end{array}$ 
Iteration $V_{\mathrm{r}}$ vs $K_{\mathrm{MPPA}} V_{\mathrm{r}}$ vs $K_{\mathrm{ACE}} K_{\mathrm{MPPA}}$ vs $K_{\mathrm{ACE}}$

Table 4 Parameter ranges used to estimate the likelihood confidence region.

\begin{tabular}{lccl}
\multicolumn{5}{l}{ Parameter Minimum Maximum Units } \\
$V_{\mathrm{r}}$ & 10 & 40 & $\mathrm{nmol} /($ Umin $)$ \\
$K_{\mathrm{ACE}}$ & 100 & 500 & $\mathrm{mM}$ \\
$K_{\mathrm{MPPA}}$ & 0.5 & 6.5 & $\mathrm{mM}$
\end{tabular}

Table 5 Parameter 95\% confidence intervals $(\mathrm{Cl})$ when using the uniform design strategy of Figure 8 . The parameter $95 \%$ Cls for the iterative rMbOED are shown in the last column (Table 2).

Parameter Value $95 \% \mathrm{Cl} 95 \% \mathrm{Cl}$

Uniform rMbOED Units

$\begin{array}{lllll}V_{\mathrm{r}} & 21.7 & \pm 11.6 & 5.2 & \mathrm{nmol} /(\text { Umin }) \\ K_{\text {ACE }} & 250 & \pm 155 & 80 & \mathrm{mM} \\ K_{\text {MPPA }} & 2.96 & \pm 2.92 & 1.49 & \mathrm{mM}\end{array}$

Table 6 Calibrated parameter values and corresponding 95\% confidence intervals for the model in Equation 2.

Parameter type Parameter Value $95 \% \mathrm{Cl}$ Units

$\begin{array}{lllll}\text { Forward } & V_{\mathrm{f}} & 24.0 & \pm 2.4 & \mathrm{nmol} /(\text { Umin) } \\ & K_{\mathrm{BA}} & 3.39 & \pm 0.63 & \mathrm{mM} \\ & K_{\text {IPA }} & 138 & \pm 22 & \mathrm{mM} \\ \text { Backward } & V_{\mathrm{r}} & 21.7 & \pm 5.2 & \mathrm{nmol} / \text { (Umin) } \\ & K_{\text {MPPA }} & 2.96 & \pm 1.49 & \mathrm{mM} \\ & K_{\text {ACE }} & 250 & \pm 80 & \mathrm{mM}\end{array}$




\section{Parameter type Parameter Value $95 \% \mathrm{Cl}$ Units}

$\begin{array}{lllll}\text { Dissociation } & K_{\text {ilPA }} & 1.76 & \pm 0.29 & \mathrm{mM} \\ & K_{\text {iMPPA }} & 1.60 & \pm 0.40 & \mathrm{mM}\end{array}$

Equilibrium $\quad K_{\text {eq }} \quad 1.94 \pm 0.71^{*}$ -

* Using Equation 3, the uncertainty of $K_{\text {eq }}$ was estimated directly from the uncertainties of the other parameters, taking into account the correlation between the different parameters.

Table 7 The correlation matrix for all the parameters from Equation 2. It can be seen that the correlation between parameters which are estimated simultaneously is high. Parameter estimates which have been estimated separately have no correlation (since they were estimated from different data sets), and are therefore set to zero.

\begin{tabular}{|c|c|c|c|c|c|c|c|}
\hline$V_{\mathrm{f}}$ & $K_{\mathrm{BA}}$ & $V_{\mathrm{IPA}} V_{\mathrm{r}}$ & & $K_{\mathrm{MPPA}}$ & $K_{\mathrm{ACE}}$ & $K_{\text {iII }}$ & $K_{\text {iMPPA }}$ \\
\hline$V_{\mathrm{f}}$ & {$\left[\begin{array}{ll}1 & 0.88\end{array}\right.$} & 0.90 & 0 & 0 & 0 & 0 & $-0.29]$ \\
\hline$K_{\mathrm{BA}}$ & -1 & 0.70 & 0 & 0 & 0 & 0 & -0.32 \\
\hline$K_{\mathrm{IPA}}$ & - & 1 & 0 & 0 & 0 & 0 & -0.31 \\
\hline$V_{\mathrm{r}}$ & - & - & 1 & 0.97 & 0.86 & 0 & 0.71 \\
\hline$K_{\mathrm{MPPA}}$ & - & - & - & 1 & 0.76 & 0 & 0.91 \\
\hline$K_{\mathrm{ACE}}$ & - & - & - & - & 1 & 0 & 0.64 \\
\hline$K_{\mathrm{iPA}}$ & - & - & - & - & - & 1 & 0 \\
\hline$K_{\mathrm{iMPPA}}$ & - & - & - & - & - & - & 0 \\
\hline$K_{\text {eq }}$ & L- - & - & - & - & - & - & 1 \\
\hline
\end{tabular}




\section{Computer}

\section{Perform initial experiment(s)}

\section{Perform} experiment

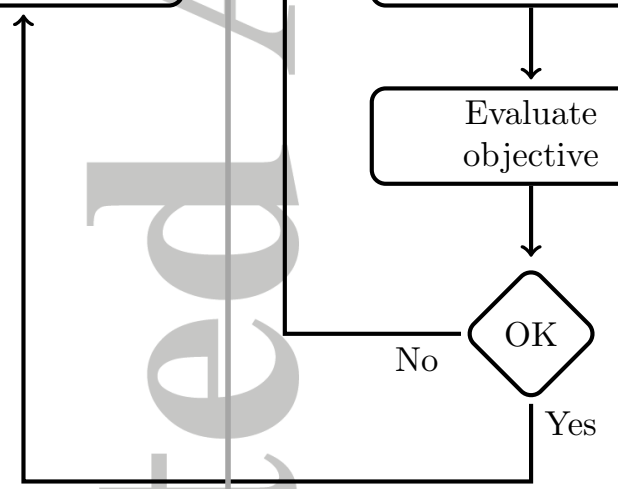

This article is protected by copyright. All rights reserved. 


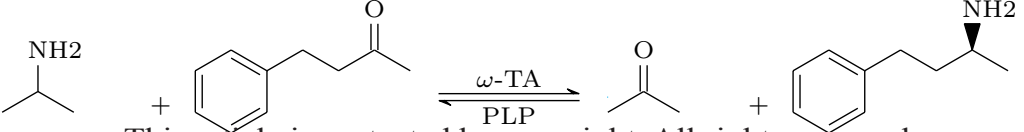
isopropylamine This article is protected by copyright All rights reserved.
IPA BA $\mathrm{ACE}$ MPPA 
$\frac{\partial v}{\partial V_{\mathrm{r}}} \frac{V_{\mathrm{r}}}{1}$

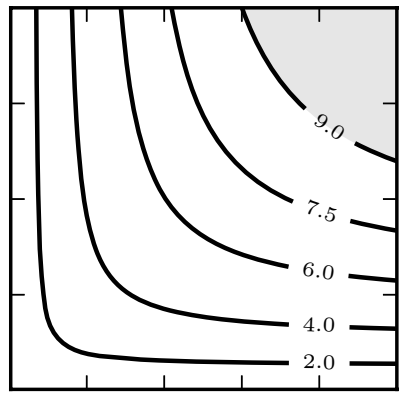

50100 Th5 10 arfeg $[A C E][\mathrm{mM}]$ $\frac{\partial v}{\partial K_{\mathrm{MPPA}}} \frac{K_{\mathrm{MPPA}}}{1}$
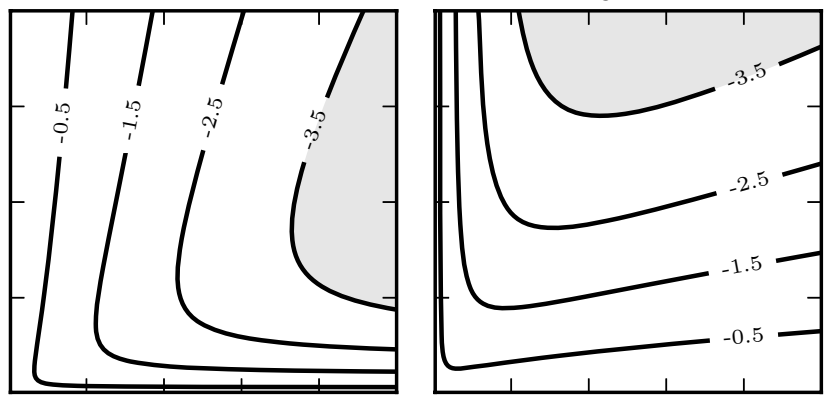

0
$259 r 8$ tectél

(l)

a by 180 pyepght. 2991 rig
$[A C E][\mathrm{mM}]$
Ats rese pled.100 $150 \quad 200 \quad 250$ $[A C E][\mathrm{mM}]$ 
$\frac{\partial v}{\partial V_{\mathrm{r}}} \frac{V_{\mathrm{r}}}{1}$

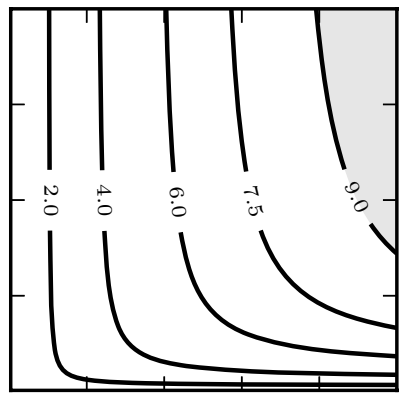

50
100 $[A C E][\mathrm{mM}]$ $\frac{\partial v}{\partial K_{\mathrm{MPPA}}} \frac{K_{\mathrm{MPPA}}}{1}$

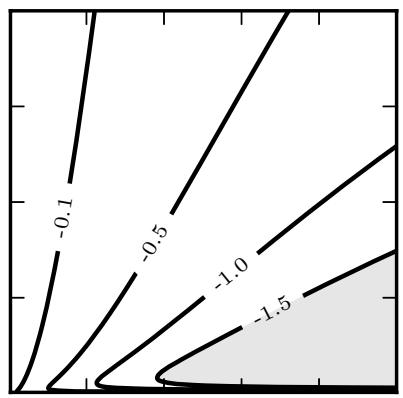

$\frac{\partial v}{\partial K_{\mathrm{ACE}}} \frac{K_{\mathrm{ACE}}}{1}$

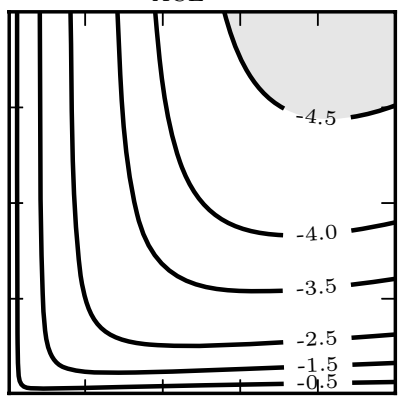

$200 \quad 250$

250 $[A C E][\mathrm{mM}]$ 


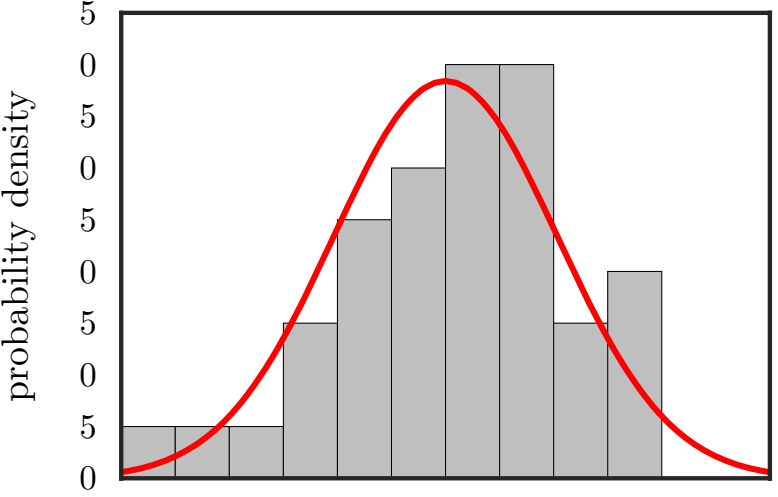

This artidie is protected by copyright. Alt rights reserved. $\epsilon_{\mathrm{rel}, i}$ 


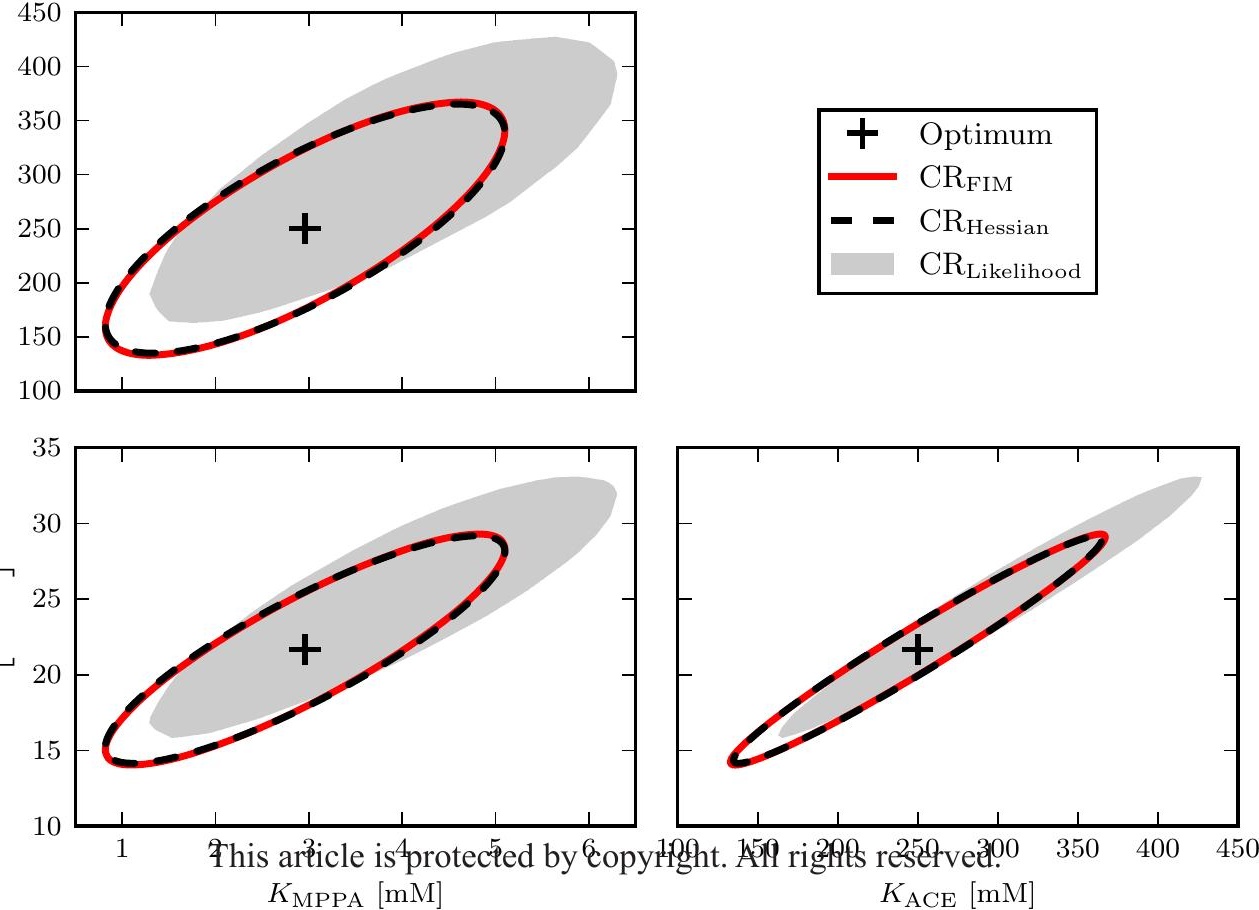




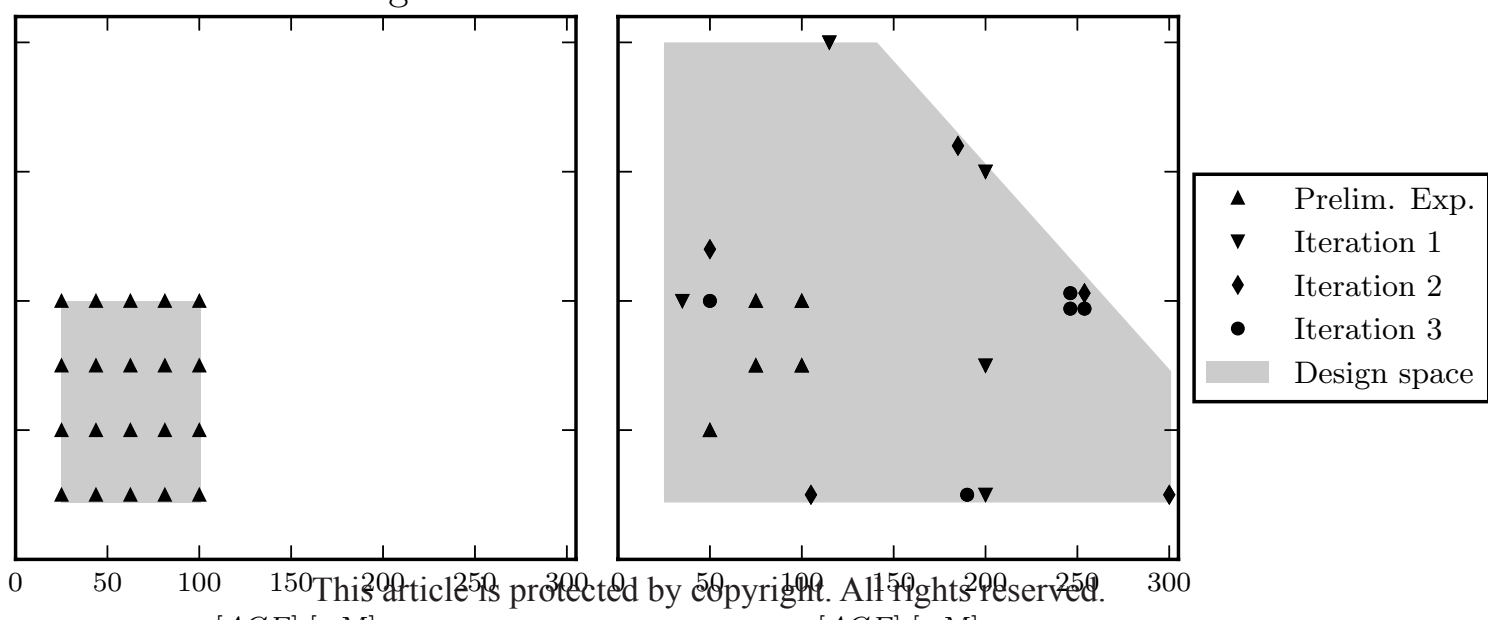
$[A C E][\mathrm{mM}]$ $[A C E][\mathrm{mM}]$ 


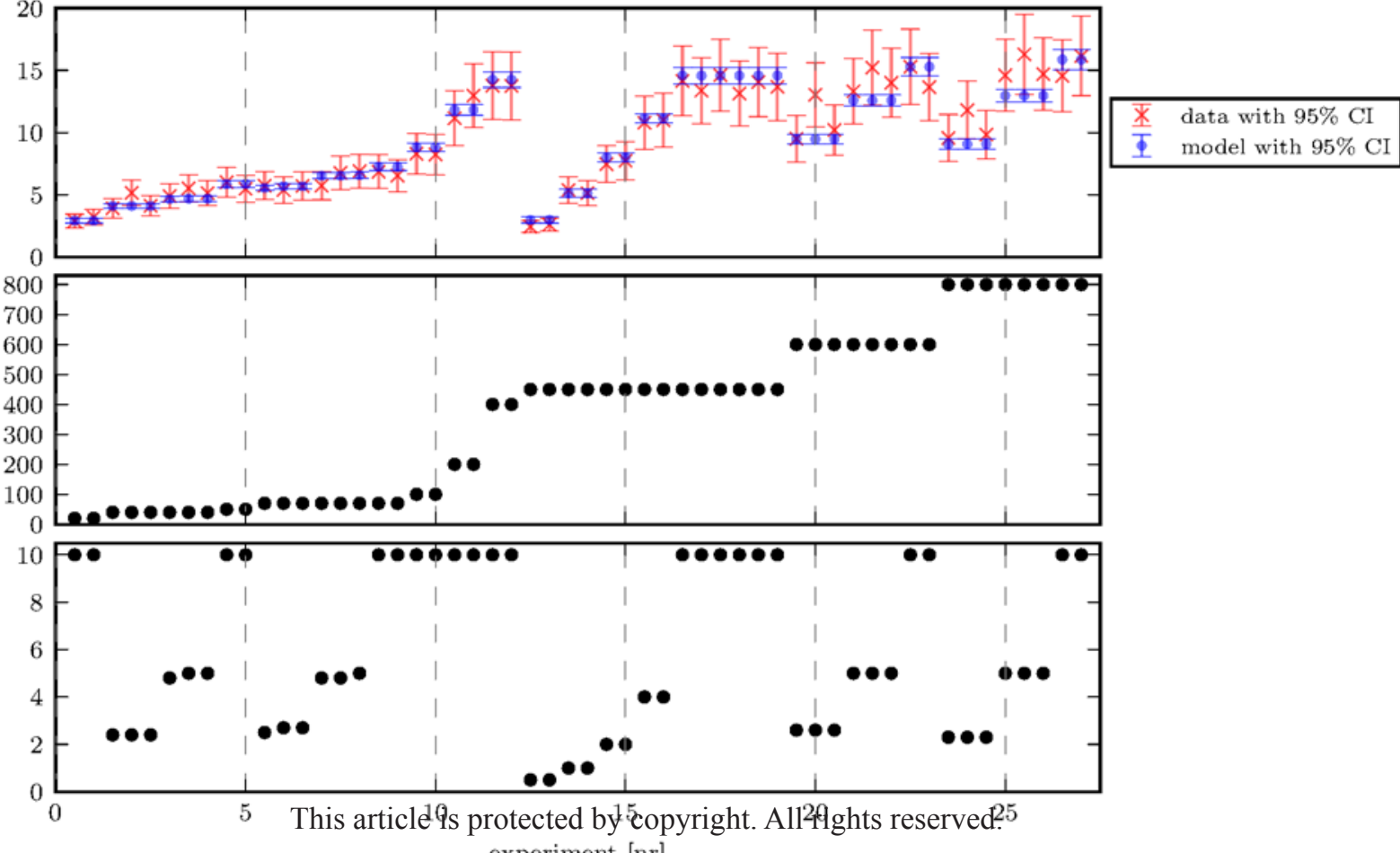




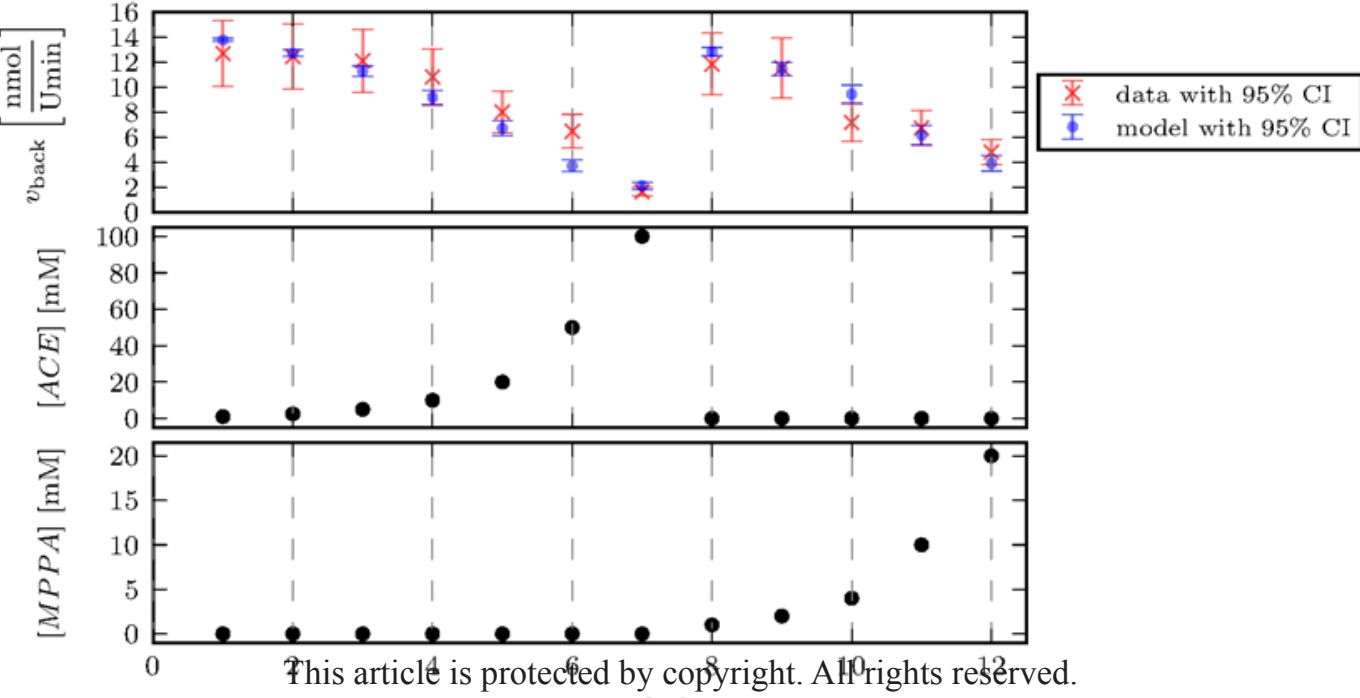
experiment [nr] 